\title{
EL DESARROLLO DE LOS RECURSOS HUMANOS Y EL CRECIMIENTO ECONÓMICO EN EL ESTADO DE JALISCO *
}

\author{
Michael C. BIRD \\ Universidad de Colorado
}

\section{INTRODUCCIÓN}

EsTa MONOGRAFÍa es un intento de resumir un estudio más completo del autor sobre el desarrollo de los recursos humanos en el estado de Jalisco y las relaciones de este desarrollo con el crecimiento económico de la entidad. Incluye consideraciones puramente cuantitativas de los incrementos de los recursos humanos (basados en tendencias demográficas), así como de los cambios cualitativos (basados en gran parte en el desarrollo del sistema educativo pero también comprendiendo aspectos fuera de este sistema). El tratamiento es histórico (se trazan los cambios en los últimos decenios) y de pronóstico, y se intenta mostrar qué tendencias del futuro son influidas por desarrollos presentes y qué consecuencias implican estas proyecciones. Debido a la naturaleza condensada de esta monografía, no he considerado práctico incluir advertencias y salvedades, así como gran parte de la documentación y explicaciones que respaldan los argumentos que aparecen en el trabajo original, $\mathbf{1}$ el cual fue escrito como tesis doctoral y se llevó a cabo con la participación directa del autor en un programa de desarrollo universitario de la Universidad Autónoma de Guadalajara ubicada en el estado de Jalisco.

Se pensó que este estudio tendría una importancia especial por dos razones fundamentales. Primero, porque Jalisco es una de las áreas clave en el desarrollo económico de México. Jalisco ha sido tradicionalmente el "granero" de México debiclo a su gran producción de maíz, en la cual ocupa el primer lugar; pero durante los últimos años ha crecido también rápidamente en importancia como centro industrial. En efecto, después del Distrito Federal y del área de Monterrey, Guadalajara (la capital de Jalisco) ocupa en la actualidad el tercer lugar entre las zonas industriales de la República. Por eso, se estimó que el estudio del desarrollo de los recursos humanos de Jalisco sería muy significativo debido a la importancia del desarrollo de esa entidad en la región suroeste del país de la cual es el centro y, por

* El título original de este trabajo es Human Resource Development and Economie Growth in the State of Jatisco, Mexico, y forma parte de un proyecto llevado a cabo bajo el patrocinio conjunto de la Universidad Autónoma de Guadalajara y la Universidad de Colorado.

1 Michael C. Bird, Human Resource Development and Economic Growth in the State of Jatisco, Universidad de Colorado, 1 sós. 
lo tanto, por su importancia sobresaliente en el desarrollo global de la economía mexicana.

Segundo, porque este estudio mostró la factibilidad de aplicar ciertas herramientas del análisis de los recursos humanos a un nivel subnacional -lo que es importante debido al creciente reconocimiento entre los economistas de la necesidad de desagregar tanto el análisis como la planificación del desarrollo económico, con objeto de evitar una dependencia exclusiva en los estudios a nivel nacional que con frecuencia ocultan agudas disparidades regionales. ${ }^{2}$

Un resumen de la descripción de la metodología que se acaba de mencionar aparece en el apéndice I de esta monografía.

\section{Crecimiento de la población y dE LA fuerza de trabajo en JaLisco}

Tendencias recierites. En los últimos años, Jalisco ha sido un área de crecimiento demográfico extremadamente rápido. Este crecimiento es típico de México y del resto de Centroamérica, una de las regiones caracterizadas por una de las tasas más altas, si no es que la mayor, de crecimiento de la población en el mundo. Esta tendencia ha sido el resultado de la disminución rápida de las tasas de mortalidad a niveles semejantes a los alcanzados por los países desarrollados, mientras que las tasas de natalidad se han mantenido a los altos niveles acostumbrados. En esa forma, puede verse en el cuadro 1 que la población de Jalisco creció de 1255346 en 1930 a 2443261 en 1960 -un incremento de casi el $100 \%$ en los últimos 30 años. No fue únicamente la base de la población la que creció rápidamente durante este período, sino también la tasa de incremento que de $1.2 \%$ anualmente entre 1930 y 1940 aumentó a $3.4 \%$ entre 1950 y 1960. Esta tendencia se ha mantenido en igual forma en todo el país, donde la tasa de mortalidad descendió de 26.6 por cada 1000 habitantes en 1930 a 10.4 en 1963, originando que la tasa de incremento de la población se elevara de $2 \%$ a $3.5 \%$ por año. ${ }^{3}$

La estructura por edades de la población de Jalisco es típica también de las áreas en desarrollo de todo el mundo. Dicha estructura de la población en un momento dado refleja en gran parte las tendencias pasadas de la mortalidad y la fecundidad. De este modo, la estructura por edades de la población de Jalisco se caracteriza por una alta concentración en los grupos de menor edad - una proporción mucho mayor que en los países desarrollados. En efecto, en 1960 la población menor de 15 años de edad en esta entidad era de cerca de un $46 \%$ del total. En contraste, el mismo dato en los Estados Unidos fue únicamente de $31.2 \%$ en el mismo año y en Europa de sólo $25 \% .4$ Como corolario de esta tendencia, la población entre las edades de 15 y 64 años es relativamente pequeña en las áreas en desarrollo como el estado de Jalisco, en donde en 1960 cerca del $50 \%$ de su población

2 En relación con este problema en el desarrollo de los recursos humanos en México, véase Charles Nash Myers, Education and National Development in Mexico, Princeton Industrial Relations Section, 1965.

3 Robert C. Cook, "Mexico: The Problem of People", Population Bultetin, Population Reference Bureau, Inc., Vol. 20, Núm. 7, noviembre de 1964, p. 176.

4 Ibid., p. 178. 
Cuadro 1

JaLisCO: CRECIMIENTO de LA POBLACIÓN Y URBANIZACtón, 1930-1980

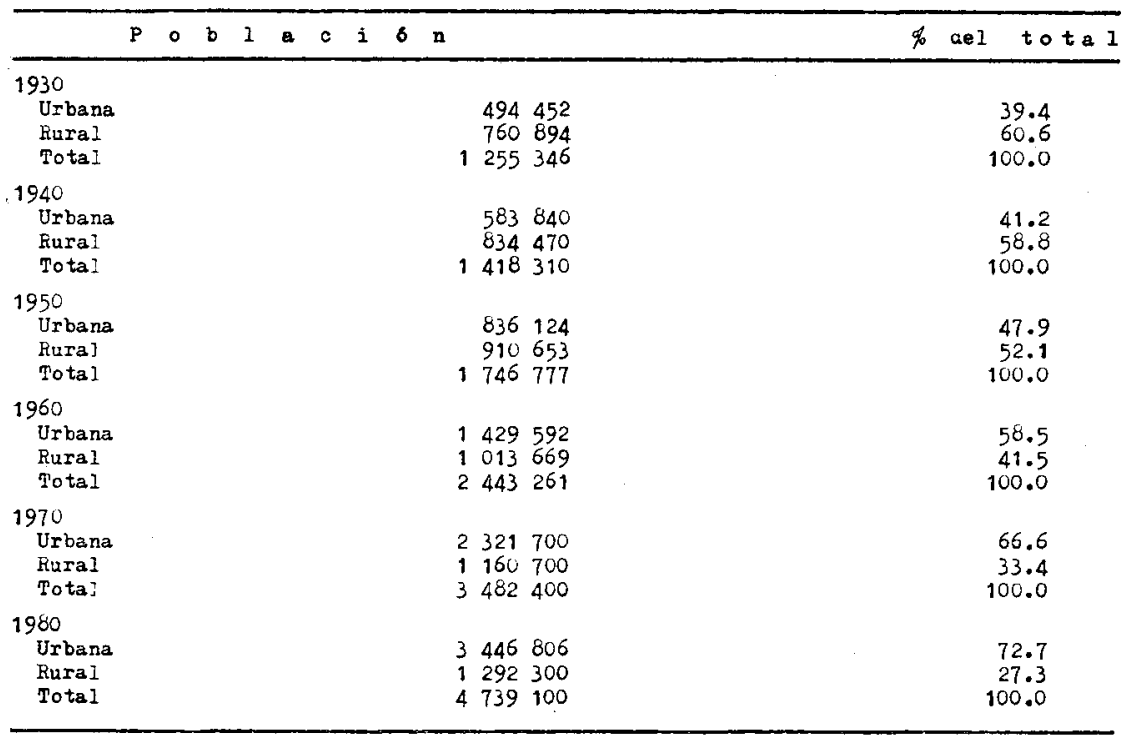

Fuentes: Los datos de población para 1930, 1940 y 1950 son del VII Censo General de población, Estado de Jalisco, México, 1953, cuadros 1, 24 y 25, pp. 2429; 218-230. Los de 1960, del VIII Censo General de Población, 1960, Resumen General, México, 1967; y para 1970 y 1980, las cifras son de Raúl Benítez Z. y Gustavo Cabrera A., Proyecciones de la pobtación de México, 1960-1980, Banco de México, 1966.

se encontraba dentro de este grupo contra un $65 \%$ en los Estados Unidos. ${ }^{5}$

Existe una significación económica de consideración en estas estructuras por edades. Donde una gran porción de la población consiste en personas no productivas en las edades inferiores a 15 y mayores de 70 años, la carga para mantener a estos grupos pesa más en los productores que se encuentran en el grupo de los 15 a los 70 años. Esta carga se hace más grave donde un porciento relativamente elevado de mujeres dentro del grupo de 15 a 70 años no son económicamente activas, como es el caso de Jalisco y en general de toda América Latina. ${ }^{6}$ Como sugieren algunas veces los demógrafos, la desventaja económica de esta situación en los países menos desarrollados no radica tanto en el número reducido de personas disponibles como fuerza de trabajo como en el efecto negativo en la propensión a ahorrar. Esto es así porque la mayoría de estos países no puede absorber productivamente la fuerza de trabajo que se encuentra disponible. También hay otras consecuencias de esta situación, como lo es la gran presión sobre el sistema educativo.

5 Louis J. Ducoff, Human Resources of Central America, Panama and Mexico, 1956-1980, en Relation to Some Aspects of Economic Development, CEPAL, México, 1960 , p. 12.

6 Ibid., p. 12. 
Urbanización. El cuadro 1 describe la aceleración del crecimiento de las áreas urbanas en Jalisco desde 1930. La población urbana aumentó su participación en la población total de la entidad de $39.4 \%$ en 1930 a $58.5 \%$ en 1960. La cifra para el último año excedió en forma significativa a la de la República, que en 1960 registró el $42.6 \%$ de su población en el área urbana. ${ }^{7}$ No solamente se incrementó la base de la población en las áreas urbanas, sino que también la tasa de urbanización se fue elevando. Entre 1930 y 1940 , la población urbana creció a una tasa anual compuesta de $1.7 \%$, mientras que entre 1950 y 1960 esta tasa creció a $5.5 \%$. Las tasas para el país en los mismos periodos fueron de $2.2 \%$ y de $4.9 \% .8$ En contraste con este modelo de crecimiento acelerado, la población rural se ha elevado a una tasa más o menos estable, variando solamente de $0.9 \%$ por año entre 1930 y 1950 a $1.1 \%$ entre 1950 y 1960 . La tasa de crecimiento de la población rural de la República se ha mantenido más o menos constante en $1.5 \%$ por año durante todo este período. ${ }^{9}$ Sin embargo, como consecuencia del mayor crecimiento de las áreas urbanas, la participación del sector rural en el total de la población del estado de Jalisco ha declinado del $60.6 \%$ en 1930 al $41.5 \%$ en 1960 . Estas tendencias son congruentes con el modelo de México y de los países centroamericanos en los últimos decenios, durante los cuales la población urbana ha crecido generalmente a una tasa anual de un 50 a un $100 \%$ más rápido que la población rural. ${ }^{10}$ Aunque las altas tasas de mortalidad y de natalidad en las áreas rurales son en parte responsables de este patrón demográfico, la emigración de las áreas rurales a las áreas urbanas se considera como el factor más importante.

La mayor parte de esta urbanización está ocurriendo en Guadalajara, la segunda ciudad más grande de la República Mexicana, donde la población ha crecido de 380000 habitantes en 1950 a una estimada en 1123000 en 1965, lo que representa un crecimiento de cerca de un $7.6 \%$ anual. Durante este periodo, Guadalajara ha incrementado su participación relativa en la población del estado de un $22 \%$ a un $38 \%$. En el presente, se estima que la población de Guadalajara seguirá creciendo a una tasa aproximada de $8 \%$ anual. Con base en información de estadisticas vitales obtenida de la Oficina de Estudios Económicos del Estado de Jalisco, se ha estimado que únicamente el $2.6 \%$ de este crecimiento es atribuible al crecimiento natural, mientras que el otro $5.4 \%$ se debe al movimiento continuo del campo hacia la ciudad. Si la tasa de crecimiento observada entre 1960 y 1965 continúa, Guadalajara tendrá alrededor de un millón y medio de habitantes en 1970 y un poco más de tres millones en 1980, lo que representa un incremento de cerca de $800 \%$ en un período de 30 años a partir de 1950. Debe notarse que esta casi increíble expansión no es única en Guadalajara. Por ejemplo, estimaciones recientes han situado a la población de la ciudad de México a la altura de 15 millones de

7 Calculado con datos del Anuario Estadístico Compendiado, 1964, SIC, Dirección General de Estadística, México, 1965, cuadros 2 y 3, p. 18.

8 Raúl Benítez Zenteno y Gustavo Cabrera Acevedo, Proyecciones de la población de México, 1960-1980, Banco de México, México, 1966, cuadro 17, p. 57.

9 Ibid., p. 57.

10 Ducoff, op. cit., p. 15. 
habitantes para 1975, o entre 7.5 y 8.5 millones sobre su nivel presente. Además, la tasa de $95 \%$ de incremento de la población de Guadalajara entre 1950 y 1960 fue solamente la cuarta más rápida entre las de las áreas urbanas más grandes de la República. Sin embargo, el aumento absoluto de 360000 ocupó el segundo lugar, después de la ciudad de México.

Población futura. El excelente estudio hecho recientemente por dos demógrafos mexicanos, Raúl Benítez Zenteno y Gustavo Cabrera, proporciona proyecciones muy significativas con respecto a las perspectivas futuras de la población de Jalisco. En su estudio se revisan cuidadosamente las proyecciones pasadas de las tendencias demográficas mexicanas, se hacen supuestos y proyecciones nuevas usando el método de componentes, presentando proyecciones por separado para cada grupo de edad y sexo y de acuerdo con la estructura rural-urbana de la población. En términos generales, suponen que la tasa de natalidad en Jalisco empezará a descender entre 1965 y 1980 (5\%, $10 \%$ y $15 \%$ respectivamente, por períodos de 5 años) y que tendrá lugar un aumento gradual en la esperanza de vida. Sus supuestos respecto a la migración resultan en una afluencia neta a Jalisco de otros estados de la República entre 1960 y 1980 . El resultado de sus hipótesis con relación a nacimientos, muertes y movimientos migratorios para la tasa de crecimiento de la población de Jalisco se muestran en el cuadro 2. (El tamaño de la población proyectado en 1970 y 1980 se mostró en el cuadro 1.)

\section{Cuadro 2}

Jalisco: Tasas de CREcimiento DE LA población URBANa Y RURAL, 1930-1960, Y PROYECCIONES, 1960-1980

(Porcientos)

\begin{tabular}{lccccc}
\hline & $1930-1940$ & $1940-1950$ & $1950-1960$ & $1960-1970$ & $1970-1980$ \\
\hline Población total & 1.2 & 2.1 & 3.4 & 3.3 & 3.1 \\
Población urbana & 1.7 & 3.6 & 5.5 & 4.7 & 4.0 \\
Población rural & 0.9 & 0.9 & 1.1 & 1.1 & 1.1 \\
\hline
\end{tabular}

Fuentes: Tasas de crecimiento de 1930-1940, 1940-1950 y 1950-1960, calculadas con cifras del cuadro 1. Las tasas de incremento de 1960-1970 y 1970-1980, fueron calculadas con cifras de Benítez y Cabrera, op. cit., cuadro 45, pp. 146-147.

El crecimiento de la población total de Jalisco entre 1960 y 1980 se estima en alrededor de 2200000 personas, o sea un incremento de $89 \%$ en 20 años. Este crecimiento implica una ligera disminución de la tasa de crecimiento anual entre 1960 y 1970 a $3.3 \%$ por año (contra $3.4 \%$ anual entre 1950 y 1960). Las proyecciones implican también un moderado retraso de la tasa de urbanización desde 1960 hasta 1980, principalmente como consecuencia de una disminución gradual supuesta de la fecundidad durante este período. Aun sobre estas bases, el crecimiento urbano está proyectado para absorber alrededor del $89 \%$ del incremento total de la población durante el período de 20 años. La composición por edades de la población se proyecta con cambios 
ligeros entre 1960 y 1980 -sólo un descenso más prolongado de la fecundidad permitiría que esta estructura se acercara más estrechamente a las de las áreas más desarrolladas del mundo, o sea con menor concentración en los grupos de menor edad.

En resumen, la población de Jalisco ha crecido rápidamente en los últimos decenios y sin duda continuará haciéndolo hasta 1980. Este crecimiento rápido y el efecto resultante de la composición por edades, de la densidad y de la estructura rural-urbana de la población, serán una condición preeminente en el trazo del desarrollo de los recursos humanos y del desarrollo socioeconómica general de Jalisco en los próximos años. El supuesto con respecto a la tendencia futura de la fecundidad, que es la variable clave en las proyecciones de la población usadas en este estudio, debe ser descrito como optimista en vista de la reciente historia demográfica mexicana. Por eso, cualquier apreciación de los problemas económicos y sociales implicados en el proyectado crecimiento y la estructura de la población deberá tomar en cuenta que las dificultades se agravarán en la medida en que este supuesto optimista no se realice. Sin embargo, el tamaño de la fuerza de trabajo durante el período previsto no será afectado directamente por los cambios en la fecundidad. A largo plazo esta variable aparece también altamente dependiente del curso de los patrones de fecundidad.

El crecimiento de la fuerza de trabajo está, por supuesto, íntimamente relacionado con el crecimiento de la población. De hecho, estableciendo las tasas de participación de la fuerza de trabajo por grupos de sexo y de edad (la proporción en cada uno de los grupos de edad que son miembros de la fuerza de trabajo), puede estimarse fácilmente el tamaño de la fuerza de trabajo futura. Estas tasas fueron determinadas en el estudio original y, cuando se aplicaron a las proyecciones de población de Benítez y Cabrera, permitieron calcular aproximadamente el tamaño futuro de la fuerza de trabajo de Jalisco. En general, el resultado global de estas tasas de participación de la fuerza de trabajo es una reducción en la proporción de hombres económicamente activos, lo que refleja incrementos en las oportunidades educativas, $y$ un ligero aumento en la proporción de las mujeres activas, que refleja grandes oportunidades para su empleo.

La aplicación de las tasas de participación resulta en un crecimiento proyectado de la fuerza de trabajo de $92 \%$ entre 1960 y 1980 , que es considerablemente más grande que el incremento del $42 \%$ entre 1930 y 1950 pero sólo ligeramente mayor que el de $89 \%$ entre 1940 y 1960. Sin embargo, el incremento absoluto de la fuerza de trabajo en el último período será mayor que en los anteriores, resultado de la expansión rápida de la base de la población. Como se muestra en el cuadro 3, se espera que el incremento anual de la fuerza de trabajo llegue a 38600 entre 1970 y 1980. Esta cifra da una aproximación burda del número anual de nuevos empleos que la economía de Jalisco necesita generar para evitar aumentos en el desempleo o el subempleo hasta 1980. Esto no considera la creación de empleos para absorber a los desocupados o subempleados que existen actualmente. Proveer empleos para incrementos anuales de esta magnitud indiscutiblemente requerirá un intenso esfuerzo de ampliación de la capacidad y los recursos públicos y privados. 
Cuadro 3

JaLisCo: Población ECONÓMICAMENTE ACTIVA, 1930-1960, y PROYeCCIONES, 1960-1980

\begin{tabular}{ccc}
\hline Periodo & $\begin{array}{c}\text { Promedio de } \\
\text { incremento anual } \\
\text { ne to }\end{array}$ & $\begin{array}{c}\text { Tasa do } \\
\text { incremento } \\
\%\end{array}$ \\
\hline $1930-1940$ & 4536 & 1.10 \\
$1940-1950$ & 11871 & 2.40 \\
$1950-1960$ & 20129 & 3.10 \\
$1960-1970$ & 26283 & 3.00 \\
$1970-1980$ & 38602 & 3.23 \\
\hline
\end{tabular}

Fuentes: Cifras para 1930-1940 y 1940-1950 calculadas con datos del VII Censo General de Población, junio de 1950, Estado de Jalisco, Dirección General de Estadística, cuadros $1,9,23$ y 24, pp. 24-29, 120-125, 218-223 y 224-229. Las cifras para 1950-1960, calculadas con datos de VIII Censo General de Población, 1960, Resumen General, Dirección General de Estadística, cuadro 8, pp. 94111; y VIII Censo General de Población, población económicamente activa, rectificación a los cuadros 25, 26 y 27, Dirección General de Estadística, cuadro 27, pp. 53-74. Por último, las cifras para 1960-1970 y 1970-1980 se calcularon con datos de los cuadros 4.7 y 4.13 del estudio original del autor.

\section{El PATRÓN FUTURo DE EMPLEO POR ACTIVIDAD ECONÓMICA}

Para relacionar las proyecciones de la población y del tamaño de la fuerza de trabajo y su composición con los requerimientos de mano de obra en la economía de Jalisco, es necesario examinar el modo en que ha evolucionado la economía de la entidad en el pasado y cómo evolucionará en el futuro. Estas necesidades de mano de obra serán, en cierto modo, función de la cantidad y la calidad de la mano de obra disponible, y constituirán a la vez la base de la demanda de esta mano de obra. Esta última consideración se pondrá de relieve en lo que sigue.

Los resultados del análisis de los patrones de empleo se muestran en el cuadro 4. Las cifras proyectadas para 1970 y 1980 se obtuvieron mediante el procedimiento descrito brevemente en el apéndice I. De nuevo, debe señalarse que este resumen de los resultados de las proyecciones está basado en el examen detallado de los sectores individuales (lo cual se describe en el estudio original completo). Aun cuando se hicieron concesiones explícitas sobre el desempleo en las proyecciones (considerando un desempleo supuesto del $3 \%$ ), este problema es incuestionablemente de menor importancia cuantitativa que el del subempleo - personas clasificadas como empleadas pero con una productividad extremadamente baja o igual a cero. Como las medidas de compensación del desempleo son inadecuadas en Jalisco (como en todo México), muchas de las personas que de otro modo podrían aparecer desempleadas son absorbidas en trabajos de muy baja productividad, por lo regular en los sectores de agricultura y de servicios, respectivamente. En el estudio, las metas del empleo implican un esfuerzo para absorber casi todos los nuevos incrementos de la fuerza de trabajo en los años futuros, sin reducir los niveles medios de productividad, más que un esfuerzo por eliminar la exïstencia actual del subempleo.

Como puede verse en el cuadro 4, de 1960 a 1980 se espera que el 
empleo primario continúe perdiendo su posición relativa en cuanto al empleo total en una forma más o menos rápida, mientras que al mismo tiempo se mantienen tasas de crecimiento absolutas anuales de un poco más de $1 \%$. El empleo en las manufacturas se proyecta con un crecimiento a una tasa absoluta de cerca de un $3 \%$, con lo que únicamente mantendrá su participación relativa en el total del empleo. En construcción crecerá a una tasa anual ligeramente mayor y aumentará su participación relativa de $4.5 \%$ a $5.8 \%$ del total. La ocupación en la minería aumentará con lentitud en términos absolutos y disminuirá ligeramente en términos relativos. El empleo en el sector eléctrico crecerá en una forma más o menos rápida e incrementará ligeramente su participación relativa. Tanto en el comercio como en los servicios, se espera que crecerá en forma rápida e incrementará considerablemente su participación relativa. El sector transportes y comunicaciones está proyectado para crecer a una tasa considerable y elevar su participación relativa muy ligeramente. En resumen, el método usado supone implícitamente que gran parte del incremento de la fuerza de trabajo durante el período de proyección será absórbido en empleos de productividad relativamente baja, en especial en el sector de servicios, más que en forma de grandes incrementos de subempleo en la agricultura. Este juicio es consonante con en el patrón de urbanización muy rápida proyectado para el estado de Jalisco.

\section{Cuadro 4}

JALISCO: EMPIEO POR SECTORES, 1950-1960, Y PROYECCIONES, 1970-1980

\begin{tabular}{|c|c|c|c|c|c|c|c|c|}
\hline \multirow{2}{*}{ Seotor } & \multicolumn{2}{|c|}{1950} & \multicolumn{2}{|c|}{1960} & \multicolumn{2}{|c|}{1970} & \multicolumn{2}{|c|}{1980} \\
\hline & Bmple0 & 9 & Empleo & $\not$ & Empleo & 8 & Emploo & 8 \\
\hline $\begin{array}{l}\text { Primario } \\
\text { Manufacturas } \\
\text { Construccion } \\
\text { Minerfa } \\
\text { Electricidad } \\
\text { Conereio } \\
\text { Transporte y }\end{array}$ & $\begin{array}{rr}324 & 660 \\
70 & 446 \\
17 & 841 \\
1 & 652 \\
1 & 755 \\
48 & 218\end{array}$ & $\begin{array}{r}58.9 \\
12.8 \\
3.2 \\
0.3 \\
0.3 \\
8.7\end{array}$ & $\begin{array}{rr}392 & 860 \\
118 & 347 \\
33 & 680 \\
4 & 784 \\
2 & 465 \\
84 & 041\end{array}$ & $\begin{array}{r}51.9 \\
15.6 \\
4.5 \\
0.6 \\
0.3 \\
11.1\end{array}$ & $\begin{array}{rr}450 & 900 \\
158 & 431 \\
53 & 970 \\
5 & 077 \\
4 & 156 \\
139 & 081\end{array}$ & $\begin{array}{r}45.3 \\
15.9 \\
5.4 \\
0.5 \\
0.4 \\
14.0\end{array}$ & $\begin{array}{rr}508 & 477 \\
217 & 880 \\
79 & 138 \\
5 & 624 \\
7 & 068 \\
232 & 898\end{array}$ & $\begin{array}{r}37.1 \\
15.9 \\
5.8 \\
0.4 \\
0.5 \\
17.0\end{array}$ \\
\hline $\begin{array}{l}\text { comunicaciones } \\
\text { Servicios } \\
\text { Total }\end{array}$ & $\begin{array}{r}13366 \\
74029 \\
551987\end{array}$ & $\begin{array}{r}2.4 \\
13.4 \\
100.0\end{array}$ & $\begin{array}{r}24712 \\
95720 \\
756 \quad 609\end{array}$ & $\begin{array}{r}3.3 \\
12.7 \\
100.0\end{array}$ & $\begin{array}{rr}34 & 277 \\
149 & 291 \\
995 & 183\end{array}$ & $\begin{array}{r}3.4 \\
15.0 \\
100.0\end{array}$ & $\begin{array}{r}57079 \\
261448 \\
1369612\end{array}$ & $\begin{array}{r}4.2 \\
19.1 \\
100.0\end{array}$ \\
\hline
\end{tabular}

Tasas anuales de crecimiento

\begin{tabular}{lccc}
\hline & $1950-1960$ & $1960-1970$ & $1970-1980$ \\
\hline Primario & 1.9 & 1.4 & 1.2 \\
Manufacturas & 5.3 & 3.0 & 3.2 \\
Construcció & 6.6 & 4.8 & 3.7 \\
Minerfa & 11.2 & 0.6 & 1.0 \\
Blectricidad & 3.5 & 5.4 & 5.4 \\
Comercio & 5.7 & 5.2 & 5.3 \\
Transporte y & & 3.3 & 5.2 \\
comunicaciones & 6.4 & 4.6 & 5.8 \\
Servielos & 2.6 & 2.8 & 3.3 \\
& 3.2 & & \\
\hline
\end{tabular}

Más que un intento de predecir el futuro, las proyecciones de empleo alcanzadas deben ser tomadas como el reflejo de un conjunto de metas económicas realistas y generalmente deseables. Estas proyec- 
ciones constituyen uno de los pasos necesarios en la formulación de un programa congruente de desarrolo de los recursos humanos. Por fortuna, para propósitos de la construcción de este programa, unos pocos lineamientos generales sobre los requerimientos de mano de obra pueden ser útiles $\mathrm{y}$, por eso, aun desviaciones considerables en los patrones del futuro crecimiento económico de las proyecciones no invalidan necesariamente el proyecto.

\section{El SISTEMA EDUCATIVO DE JALISCO}

Advertencias introductorias. Antes de relacionar las tendencias pro. yectadas del empleo en Jalisco con los requerimientos de fuerza de trabajo implícitos, es necesario analizar y describir el desarrollo de los recursos humanos en Jalisco hasta el presente, o al menos hasta un pasado reciente. Este análisis se concentra en el desarrollo del sistema de educación formal en la entidad pero también considera aspectos fuera de este sistema, especialmente en cuanto al adiestramiento en el trabajo. Una vez más, este pequeño resumen únicamente toma en cuenta algunos datos pertinentes del examen más detallado hecho en el estudio original. En éste y en otros trabajos se encontrará también una explicación más completa de la estructura y la organización administrativa del sistema educativo.

El sistema de educación en México tiene tres divisiones principales : primaria, secundaria y educación superior. En los párrafos que siguen se trata el desarrollo de cada una de éstas en Jalisco y se hacen algunas comparaciones con otras áreas de la República.

En este punto es quizás apropiado mencionar brevemente el estado presente del pensamiento en México en relación al papel de la educación en el desarrollo social, económico y cultural de la nación. Parece muy claro que los dirigentes del país han llegado a reconocer a la educación como una herramienta básica que debe ser usada para implementar el desarrollo económico. ${ }^{11}$ La siguiente transcripción del texto de un discurso del presidente Díaz Ordaz indica el grado hasta el cual ha evolucionado esta nueva urgencia sobre el contenido funcional y práctico de la educación:

Para el México contemporáneo es de vital importancia que la educación esté orientada hacia el trabajo productivo. Necesitamos el desarrollo rápido de todo tipo de personal, desde el científico de alto nivel hasta el trabajador semicalificado que México necesita. Los requerimientos de fuerza de trabajo de alto nivel están aumentando más rápidamente que la fuerza de trabajo en general. Buscaremos ligar los planes educacionales a las políticas de empleo para equilibrar la oferta y la demanda de fuerza de trabajo calificada y semicalificada. 12

Esto está muy lejos de ser el concepto clásico, elitista, de educación que prevaleció durante la mayor parte del período prerrevolu-

11 Véase Marjorie C. Johnston, Education in Mexico, U. S. Department of Health, Education and Welfare, Washington, D.C., 1966, y La educación priblica en México, Secretaría de Educación Pública, Vol. I, Núm. 9, 1966. p. 35 .

12 Tomado de un discurso citado en La educación pública en México, op. cit., 
cionario. Por eso, el problema no es ahora el de fomentar un modo más dinámico de pensamiento sobre el papel de la educación, sino de llevar a cabo estudios empíricos, particularmente a nivel regional, necesarios para implementar el nuevo modo de pensar acerca de la educación y el desarrollo de los recursos humanos.

En áreas de rápido crecimiento de población como Jalisco, una elevada proporción de la población está concentrada en los grupos de edad escolar. El cuadro 5 indica el aumento en los grupos de edad escolar correspondiente a las principales divisiones educativas entre 1950 y 1960 . Se observa que la población de Jalisco aumentó en cerca de 700000 entre 1950 y 1960, y que cerca del $60 \%$ de este incremento se centró en los grupos de edad escolar. Dividiendo este incremento por grupos de edad, el grupo de 6-14 ha experimentado un aumento de cerca de 200000 , o sea del $50 \%$; el grupo de 15-19 un incremento de cerca de 80000 , o un poco menos de $50 \%$; y el de 20-29 ha tenido una expansión de cerca de 75000 , que equivale a un $27 \%$. El total de la población en edad escolar (incluyendo los niños en edad preprimaria) creció en cerca de 400000 , o $43 \%$ sobre el nivel de 1950. Esta tendencia de rápido crecimiento (absoluto y relativo) de la población en edad escolar continuará a través del período previsto y constituirá una carga muy considerable sobre la capacidad educativa del estado de Jalisco.

\section{Cuadro 5}

JaLisoo: Crecimiento de La poblactón en edad escolar, 1950-1960

\begin{tabular}{lcccc}
\hline & 1950 & 1960 & $\begin{array}{c}\text { \% de } \\
\text { incremento }\end{array}$ & $\begin{array}{c}\text { Tasa de } \\
\text { incremento }\end{array}$ \\
\hline Edad pre-escolar (4-5) & 106780 & 159243 & 49.1 & 4.1 \\
Edad primaria (6-14) & 404468 & 605693 & 49.8 & 4.1 \\
Edad intermedia (15-19) & 171561 & 253316 & 47.1 & 4.0 \\
Educacion superior (20-29) & 276220 & 349569 & 26.6 & 2.4 \\
Poblacion escolar total & 959029 & 1367821 & 42.5 & 3.6 \\
Poblacion & 1746777 & 2443261 & 39.9 & 3.4 \\
\hline
\end{tabular}

Fuentes: Cifras para 1950, de datos del VII Censo de Población, junio de 1950, Estado de Jalisco, Dirección General de Estadística, cuadro 3, pp. 48-89. Para 1960, cifras con base en el VIII Censo General de Población, 1960. Resumen General, cuadros 7 y 27 , pp. $85-93$ y 434460 .

Educación primaria. El cuadro 6 describe el crecimiento del grupo de 6 a 14 años de edad, el aumento de las inscripciones de este grupo en la educación primaria y el porciento de inscripciones en Jalisco de 1950 a 1960. Es probable que el crecimiento del grupo de edad de 6 a 14 años y las cifras de inscripción hayan sido subestimadas entre 1952 y 1959 . Esto se revela por las cifras presumiblemente más exactas para 1960, probablemente derivadas del censo de población de ese año. De este modo, el aparente crecimiento continuo de las tasas de inscripción en Jalisco probablemente no existió. Por lo tanto, la comparación más significativa se hace entre 1952 y 1960 , omitiendo los años intermedios. Sobre esta base puede verse que la tasa de crecimiento de las inscripciones en Jalisco no alcanzó, por margen muy estrecho, a mantenerse a la par con la tasa de crecimiento 
del grupo de edad de 6-14, lo que originó una muy ligera declinación en la tasa de inscripción de este grupo de edad. Por otro lado, en el estado de Nuevo León y en la República entera se han registrado tasas de crecimiento de las inscripciones que exceden las tasas de crecimiento del grupo de 6 a 14 años, resultando en una elevación de las tasas de inscripción. Como la tasa de inscripción en Nuevo León excedía ya a la de Jalisco en 1952 ( $66.5 \%$ contra 52.2\%), la diferencia se incrementó por las experiencias divergentes de los dos estados en los años cincuenta. Más aún, aunque en 1952, la tasa de inscripción en Jalisco fue ligeramente mayor que la de la República para 1960 , esta situación se ha invertido y la diferencia se ha agrandado. Desde otro punto de vista, no obstante que la tasa de incremento de las inscripciones en Jalisco fue más o menos igual a la tasa de crecimiento del grupo de 6 a 14 años, el número absoluto de niños de este grupo de edad no inscritos en las escuelas primarias aumentó de cerca de 200000 en 1952 a casi 300000 en 1960. Entonces, parece claro que hacia 1960 el progreso cuantitativo general de las inscripciones en educación primaria en Jalisco no había sido satisfactorio en comparación con el resto del país.

\section{Cuadro 6}

JALISCO: CRECIMIENTO DE LA INSCRIPCIÓN EN EDUCACrÓN PRIMARIA, 1951-1960

\begin{tabular}{cccc}
\hline Años & $\begin{array}{c}\text { Poblacion de } \\
6 \text { a } 14 \text { años }\end{array}$ & $\begin{array}{c}\text { Población que } \\
\text { recibe educación }\end{array}$ & $\begin{array}{c}\text { \% que re- } \\
\text { cibe educación }\end{array}$ \\
1952 & 414333 & 216338 & 52.2 \\
1953 & 423833 & 223589 & 52.8 \\
1954 & 433524 & 230244 & 53.1 \\
1955 & 443437 & 240824 & 54.3 \\
1956 & 453576 & 24759 & 54.6 \\
1957 & 463976 & 255685 & 56.7 \\
1958 & 474585 & 26897 & 56.8 \\
1959 & 485436 & 314124 & 51.9 \\
1960 & 605693 & & \\
Tasa anual de & & & \\
increnento & & 4.8 &
\end{tabular}

Fuentes: Población y cifras de inscripción, del Anuario Estađístico, edición y referencia de páginas como sigue: 1952-1954, Anuario Estadístico, 1954, cuadro 65, pp. 155-157; 1955-1957, Anuario Estadistico 1957, cuadro 68, pp. 186-188; 1958-1959, Anuario Estadistico, 1958-1959, cuadro 85, pp. 151-153; 1960-1961, Anuario Estadistico, 1960-1961, cuadro 6.6, p. 181.

Se analizaron por separado las áreas rurales de Jalisco y se comprobó de modo similar que Jalisco se está rezagando en relación con otras entidades relativamente avanzadas como Nuevo León y Tamaulipas. En efecto, en 1960 Jalisco sólo tenía en sus escuelas rurales un porciento de inscripción de la población en edad escolar muy parecida a la de los estados más atrasados, como Zacatecas y Chiapas. Aún más, como en aquellas entidades atrasadas, una gran proporción de las inscripciones (56.8\%) en Jalisco se concentra en el primer año y una proporción muy baja $(1.1 \%)$ en el sexto grado escolar. Otros indicadores, tales como la construcción de escuelas, el aumento 
del número de maestros de instrucción primaria y el coeficiente de estudiantes por maestro, también muestran que Jalisco no ha progresado en forma rápida y suficiente en los últimos años, ya sea sobre una base absoluta, o comparativamente con otras áreas más avanzadas de la República.

Un indicador importante de la calidad educativa es la tasa de deserción escolar, debido a la reprobación o al abandono que ocurren a medida que los estudiantes van ascendiendo en la pirámide educativa. En Jalisco, la tasa de deserción escolar es mayor en los primeros dos años de educación primaria. De 1951 a 1952, apenas alrededor del $34 \%$ de los estudiantes que se inscribieron en el primer grado de instrucción primaria lograron aprobar el segundo. Aunque esta cifra se ha elevado a $44 \%$ de 1962 a 1963, en relación con la tasa nacional de $45 \%$ en estos años, indica que el sistema escolar es extremadamente ineficiente. Del tercer año en adelante el proceso de deserción disminuye; sin embargo, las pérdidas son sustanciales. De este modo, en la generación de estudiantes de 1951 a 1956 únicamente el $11 \%$ de los inscritos en el primer grado habían completado 6 años de educación primaria en 1956. La eficiencia final se incrementó hasta $20 \%$ para la generación de estudiantes de 1958 a 1963 siguiendo por un escaso margen la cifra nacional de $21 \%$.

En resumen, la educación primaria en Jalisco durante el decenio de los cincuenta y los primeros años sesenta no ha mantenido un ritmo más o menos igual al promedio logrado en otros aspectos de la economía del estado. A menos que esta situación sea mejorada, esta fase del desarrollo de los recursos humanos puede convertirse en un nudo de obstrucción, tanto para el desarrollo general de los recursos humanos como para el crecimiento económico sostenido y rápido del estado. Esta afirmación se comprueba con el análisis de los requerimientos de mano de obra, que es la parte central de este estudio. El desarrollo de la educación primaria en las áreas rurales requiere particular atención con el objeto de mitigar las discrepancias entre este sector y el sistema de educación primaria urbana. El que parte de estas inversiones en capital humano en las áreas rurales determine beneficios a las áreas urbanas, vía migración, sólo fortalece el argumento de que no debe permitirse que el desarrollo de la escuela primaria aparezca demasiado concentrado en las ciudades. De otra manera, los emigrantes no tendrán los antecedentes necesarios para permitir su absorción efectiva en la economía urbana.

El progreso relativamente lento de la educación primaria en Jalisco se refleja en el grado de alfabetismo de la población del estado. Un período de cuatro años de educación primaria es a menudo considerado como el requisito mínimo necesario para alcanzar un "alfabetismo funcional", o sea una habilidad permanente para leer y escribir. Sin embargo, se usan frecuentemente medidas alternativas de los logros en alfabetismo. Por ejemplo, los censos en México miden el alfabetismo preguntando a cada individuo censado si sabe leer o escribir. Aunque esta técnica es probablemente razonable en áreas urbanas, exagera el nivel real del alfabetismo en las áreas rurales donde un número considerable de personas ha perdido su grado de alfabetismo (el cual fue obtenido en menos de los cuatro años marcados 
como mínimo para la instrucción primaria) por la falta de uso. Se han hecho cálculos usando ambos métodos. Con la definición del censo, se ve claramente que hubo un progreso sustancial entre 1930 y 1960, cuando la proporción alfabeta cle la población de 6 años de edad o más aumentó de un $37.6 \%$ a un $65.1 \%$. Sin embargo, habŕa aún 543650 personas analfabetas en 1960. No obstante este marcado incremento, Jalisco se clasificó más o menos a la mitad entre todas las entidades de la República en 1960. Nuevo León acusó una tasa de alfabetización de $80.7 \%$, Sonora de $76.2 \%$ y la República una de $62.2 \%$.

Con base en la definición que considera como alfabetos únicamente a aquellos que han completado 4 o más años de educación formal, el panorama deja mucho que desear. De acuerdo con esta definición, únicamente el $20.4 \%$ de la población con más de 14 años en Jalisco puede considerarse como alfabeta en 1960 , y en 1965, con base en una muestra de alrededor de 2000 familias, cerca de $45.1 \%$. No obstante que esto parece extremado, sirve para recalcar que las declaraciones oficiales que reflejan las cifras del censo pueden sobrestimar considerablemente la situación real. Otro indicador del progreso limitado en el pasado, es el promedio del grado de educación de la población de más de 30 años de edad (con esto se elimina casi a todos los que aún se encuentran en la escuela). La cifra media para Jalisco en 1960 fue de 1.9 años de educación, lo cual una vez más coloca a esta entidad a la mitad de la lista de los estados de la República. En el extremo superior la cifra correspondiente a Nuevo León fue de 3.5 años y al Distrito Federal de 4.6 años, mientras que la media nacional fue de 2.3 años. Por eso el gobierno del estado de Jalisco ha sido estimulado a hacer esfuerzos muy especiales en el campo de las campañas de alfabetización tratando de que se obtenga un avance mayor que el logrado en los programas regulares de educación.

Educación media. El cuadro 7 describe el incremento de las inscripciones en educación media en el estado de Jalisco de 1958 a 1964. Hay dos ciclos en este nivel educacional. El ciclo básico de tres años comprende escuelas secundarias generales, escuelas prenormales (muy similares a las escuelas secundarias en su contenido), escuelas secundarias técnicas (similares en curriculum a las escuelas secundarias generales pero con orientación hacia las carreras técnico-científicas), escuelas comerciales (con énfasis en conocimientos de oficina), escuelas de capacitación (con cursos vocacionales de extensión variable), y escuelas especiales (una miscelánea de instituciones). El ciclo avanzado consiste de escuelas preparatorias (o bachillerato) y las técnicas medias (para el adiestramiento de un nivel más alto de técnicos), y tiene generalmente una duración de dos o tres años. Los dos ciclos han sido combinados en los cuadros debido a las ambiguiedades en algunos casos acerca de las clasificaciones correctas. Como lo muestra el cuadro, el incremento en las inscripciones se acentúa después de 1960, si bien la mayor parte de este incremento ha ocurrido en escuelas especiales, donde la inscripción fue probablemente subestimada en 1959 y 1960.

Las inscripciones en educación media en Jalisco y en otras regiones 


\section{Cuadro 7}

JALISCO: CRECIMIENTO DE LA INSCRIPCIÓN EN EL NIVEI, INTERMEDIO DE EDUCACIÓN, 1958-1964

\begin{tabular}{|c|c|c|c|c|c|c|c|}
\hline Rama & 1958 & 1959 & 1960 & 1961 & 1962 & 1963 & 1964 \\
\hline $\begin{array}{l}\text { Secundaria general } \\
\text { Pre normal } \\
\text { Secunaaria tecnica } \\
\text { Escuelas enpeciales } \\
\text { Comeroial } \\
\text { Normal } \\
\text { Preparatoria } \\
\text { Vocacional } \\
\text { Capacitacion } \\
\text { Total }\end{array}$ & $\begin{array}{rr}8 & 310 \\
& 924 \\
& \\
7 & 354 \\
1 & 237 \\
2 & 087 \\
& \text { NA } \\
& \text { NA } \\
& 682 \\
20 & 594\end{array}$ & $\begin{array}{rr}9 & 665 \\
1 & 100 \\
& -- \\
1 & 911 \\
& 434 \\
2 & 431 \\
2 & 875 \\
& 14 \\
& 656 \\
19 & 072\end{array}$ & 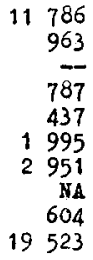 & $\begin{array}{rr}11 & 132 \\
1 & 161 \\
9 & 942 \\
9 & 598 \\
1 & 598 \\
2 & 554 \\
3 & 531 \\
\text { NA } \\
\\
& 722 \\
30 & 640\end{array}$ & $\begin{array}{rr}16 & 849 \\
1 & 100 \\
& 87 \\
10 & 068 \\
1 & 560 \\
4 & 112 \\
3 & 817 \\
& 904 \\
& 809 \\
39 & 306\end{array}$ & $\begin{array}{rr}20 & 519 \\
1 & 053 \\
& 441 \\
11 & 735 \\
& 838 \\
3 & 635 \\
4 & 862 \\
& 968 \\
& 702 \\
44 & 753\end{array}$ & $\begin{array}{rr}22 & 782 \\
1 & 083 \\
1 & 235 \\
11 & 828 \\
3 & 394 \\
3 & 384 \\
5 & 724 \\
& 992 \\
& 586 \\
51 & 008\end{array}$ \\
\hline
\end{tabular}

Fuentes: Inscripciones en secundarias general y técnica, en prenormales y centros de capacitación, de datos proporcionados por el Banco de México, Oficina de Recursos Humanos. Inscripciones en preparatorias (incluidas las preparatorias técnicas), cortesía del licenciado Adrián Lajous Vargas de El Colegio de México. Inscripciones en escuelas especiales, escuelas comerciales, normales y vocacionales del Anuario Estadístico, ediciones y referencias de página como sigue: 1958. Anuario Estadístico, 1958-1959, cuadro 104, pp. 244261; 1959-1960, Anuario Estadístico, 1960-1961, cuadros 6.54 y 6.55, pp. 238-253; 1961-1962, Anuario Estadístico, 1962-1963, cuadros 6.28 y 6.29 , pp. 220-235; 1963-1964, Anuario Estadístico, $1964-1965$, cuadros 6.26 y 6.27 , pp. $226-229$.

de México como porciento de los grupos de edad de 15 a 19 años (la mayor fuente de estudiantes a este nivel educacional) se muestra en el cuadro 8. A fin de hacer las cifras lo más comparables posible, fueron usadas las mismas fuentes para todas las entidades de la República. Sin embargo, la aplicación de información más completa sobre las inscripciones en Jalisco (dada en el cuadro 7), apenas elevaría la tasa en la entidad a un $7.7 \%$, y el uso de la cifra probablemente más acertada para 1961 resultaría en una tasa de cerca del $12 \%$, todavía menor que la tasa nacional. Por eso, Jalisco va ligeramente adelante

\section{Cuadro 8}

\section{COMPARACTÓN DE INSCRIPCTONES EN EDUCACIÓN INTERMEDIa EN JaLISCO CON OTRAS ENTTDADES DE MÉxtCo, 1960}

\begin{tabular}{|c|c|c|}
\hline Entidad & \% del grupo de edad 15-19 & 9 inscrito en educacion intermedia \\
\hline $\begin{array}{l}\text { Jalisco } \\
\text { Nuevo Ieon } \\
\text { Listrito Federal } \\
\text { Guerrero } \\
\text { Oaxaca } \\
\text { Chiapas } \\
\text { Republica }\end{array}$ & $\begin{array}{r}6.0 \\
25.4 \\
36.6 \\
3.0 \\
3.0 \\
3.0 \\
12.7\end{array}$ & $\begin{array}{l}1.72 \\
\text { (aproximado) } \\
\text { (aproximado) } \\
\text { (aproximado) }\end{array}$ \\
\hline
\end{tabular}

Fuentes: El porciento para Jalisco fue calculado usando dos métodos diferentes: 1 basado en datos del Anuario Estadístico de los Estados Unidos Mexicanos, 1960-1961, cuadro 6.55, pp. 246-253 (este método es el mismo que el usado por Myers).

2 basado en datos del cuadro 6.9, que es más exacto que el Anuario Estadístico usado por sí mismo. Porcientos para las otras entidades y para la República, de C. N. Myers, Education and National Development in Mexico, p. 58 y cuadro 9, p. 93. 
de los estados más pobres de la República como Chiapas y Guerrero en esta fase del desarrollo educativo y muy considerablemente atrás de áreas relativamente avanzadas como Nuevo León y el Distrito Federal. Las tasas de deserción escolar debidas al abandono y a la reprobación también fueron calculadas para los niveles de educación media, las cuales se encontraron muy elevadas, pero no tan altas como en los primeros tres años de la educación primaria. La razón de este alto grado de desperdicio de la educación en Jalisco, como en el resto de la República en general, obviamente debe ser investigada con sumo cuidado.

El adiestramiento vocacional en Jalisco (la información obtenida en esta área es muy deficiente) experimenta al parecer un pequeño crecimiento (véase el cuadro 7) entre 1958 y 1964 . El principal establecimiento para el entrenamiento de técnicos a nivel intermedio (esto corresponde al ciclo avanzado de la educación media) es la Escuela Politécnica de Guadalajara. Esta Escuela es parte de la Universidad de Guadalajara y está afiliada al Instituto Politécnico Nacional de la ciudad de México, la principal institución para el desarrollo de técnicos en toda la República. La escuela de Guadalajara prepara técnicos en mecánica, electricistas, técnicos en química, en electrónica y otros tipos de técnicos vitales para la industria. Junto con esto, se imparten cursos cortos para operadores de maquinaria y equipo, carpinteros, trabajadores metalúrgicos y otros. El total de la inscripción en 1963-1964 fue de 699 pero no se tiene a la mano una división de esta cifra por técnicas o cursos. Ningún entrenamiento es proporcionado por esta escuela más allá del nivel intermedio. ${ }^{13}$ La educación vocacional de los trabajadores a bajo nivel se concentra en los cursos de capacitación, que someramente corresponden al ciclo básico de educación media. La proporción de los estudiantes inscritos en estos cursos cae de un $7 \%$ del total de las inscripciones en la educación secundaria en 1958 a un $2 \%$ en 1964. No hay ningún programa para el adiestramiento de subprofesionales para la industria en Guadalajara. De hecho, este nivel de adiestramiento se limita al parecer a las escuelas de enfermería y trabajo social operadas por la Universidad de Guadalajara y la Universidad Autónoma de Guadalajara, y aún éstas no se considera que pertenecen a un nivel educativo a la par con el primer año de otras carreras profesionales.

Aunque el adiestramiento vocacional en Jalisco ha mostrado deficiencias y no ha sido suficientemente compensado por programas de entrenamiento dentro de las empresas individuales (una deficiencia que será examinada brevemente), la situación se aliviará considerablemente con la instalación del Centro Regional de Enseñanza Industrial en 1968. Este centro proporcionará preparación técnica a nivel avanzado para unos 3000 estudiantes y tendrá como propósito principal la preparación de maestros vocacionales calificados para los varios niveles de educación vocacional. Será construido con un costo de cerca de Dls. 5440000 , que deberá ser financiado por el Gobierno Federal (2960000), con una aportación del Fondo Especial de las Naciones Unidas (1520000) y el resto $(960000)$ por el estado de Jalisco.

13 Russell G. Davis, Scientific Engineering and Technical Education in Mexico, Education and World Affairs Report, Núm. 3, Nueva York, 1967, p. 13. 
Sin embargo, aunque el mencionado proyecto deberá ser un gran estímulo para el desarrollo de la mano de obra técnica en Jalisco, serán necesarios otros cambios si ha de ser enteramente satisfactorio. Estos cambios tendrán que ocurrir, por un lado, en la actitud de los ciudadanos hacia el deseo de obtener educación técnica, y por otro lado, en las prácticas de las empresas industriales en Guadalajara. En el presente, tanto en México como en Latinoamérica en general, la educación humanística-literaria es favorecida fuertemente por la mayoría de las familias. Por una parte, esto refleja un incentivo poco realista en la estructura de la industria, en la cual las escasas habilidades técnicas frecuentemente no reciben la valoración adecuada. Como resultado, los programas de adiestramiento vocacional han venido teniendo poca aceptación, prestigio y reconocimiento. La industria muy a menudo ha sido capaz de compensar la falta de técnicos en el pasado por la utilización de personal sobrecalificado o subcalificado, pero ésta no es una solución eficiente a largo plazo. ${ }^{14}$

Aunque no puede hacerse aquí una evaluación completa de la calidad de la educación media en Jalisco, parece claro que hay algunas deficiencias graves. Los administradores y los miembros del cuerpo de profesores de las universidades se quejan generalmente del bajo nivel alcanzado por los estudiantes que ingresan a los primeros años de universidad en habilidades básicas como lo son la presentación organizada de trabajos escritos y una falta considerable de habilidad para trabajar de acuerdo con las más elementales técnicas matemáticas. Por otra parte, los estudiantes son probablemente expuestos a un margen más amplio de materias que su contraparte en los Estados Unidos. Esto es consecuencia de un concepto de universidad como una institución para el entrenamiento de personas en alguna profesión, en lugar de una institución destinada a impartir un tipo de educación general diversificada como es común en los Estados Unidos. Por eso, la preparatoria llena la función de una preparación en las artes liberales - una tarea muy difícil de cumplir debido a la edad algo inmadura (15 a 17 años) de muchos estudiantes.

Educación superior. Jalisco cuenta con cuatro instituciones de educación superior: Universidad de Guadalajara, Universidad Autónoma de Guadalajara (UAG), el Instituto Tecnológico y de Estudios Superiores de Occidente (ITESo), y la Escuela Normal Superior "Nueva Galicia", las cuales, por las diferencias en sus filosofías y organizaciones educativas, proveen al estado de una considerable flexibilidad a este nivel educativo. Aunque estas instituciones han sido descritas con cierto detalle en el estudio original, las limitaciones de espacio restringen la discusión aquí a un resumen del desarrollo global de las cuatro.

El cuadro 9 indica que el total de inscripciones creció de 4656 en 1958 a 14554 en 1966. Esto representa un aumento de cerca de un $213 \%$ o una tasa anual de crecimiento de un $15 \%$. Este rápido crecimiento ha permitido que la tasa de inscripciones para el grupo de edad de 20-24 años se haya elevado de $2.7 \%$ en 1960 a $4.8 \%$ en 1965 . Sin embargo, aunque la tasa de inscripciones en Jalisco (en 1960) fue

14. Véase Davis, op. cit., p. 27-29. 
aproximadamente la misma que la de la República, se compara desfavorablemente con las tasas de Nuevo León (7.3\%) y el Distrito Federal (12.9\%). Las cifras nacionales para 1960 mostraban que Jalisco contaba con un $6.7 \%$ del total de las inscripciones en educación superior y un $6.6 \%$ de la población entre los 20 a 24 años de edad. Esto también fue comparado desfavorablemente con otras áreas avanzadas de la República: Nuevo León tiene $9.4 \%$ del total de las inscripciones con únicamente $3.4 \%$ de la población pertinente y el Distrito Federal el $73.1 \%$ con el $15.1 \%$, respectivamente.

\section{Cuadro 9}

JALISCO: INSCRTPCIONES EN EDUCACTÓN SUPERTOR, POR INSTITUCIÓN, 1958-1966

\begin{tabular}{|c|c|c|c|c|c|c|c|c|c|c|}
\hline Institucion & 1958 & 1959 & 1960 & 1961 & 1962 & 1963 & 1964 & 1965 & 1966 & $\begin{array}{c}\text { do } \\
\text { incremento } \\
1958-1966 \\
\end{array}$ \\
\hline $\begin{array}{l}\text { Universidad de Guade } \\
\text { lajara }\end{array}$ & 2998 & 3075 & 3245 & 3872 & 4842 & 5324 & 6512 & 3025 & 10315 & 244.1 \\
\hline $\begin{array}{l}\text { Universidad Autbroma } \\
\text { de Guadalajara }\end{array}$ & 1427 & 1637 & 1766 & 1567 & 2004 & 2313 & 2618 & 3021 & 3512 & 146.1 \\
\hline ITESO & 100 & 147 & 150 & 187 & 218 & 234 & 232 & 387 & 414 & 314.0 \\
\hline $\begin{array}{l}\text { Normal Superior } \\
\text { "Nueva Calicia" }\end{array}$ & 130 & 146 & 144 & 136 & 237 & 286 & 286 & 348 & 313 & 140.8 \\
\hline Total & 4655 & 5005 & 5305 & 5762 & 7307 & 8157 & 9648 & 11781 & 14554 & 212.7 \\
\hline
\end{tabular}

Fuentes: Datos sobre la Universidad de Guadalajara para 1958-1965 de La educación superior en México, 1966, Asociación Nacional de Universidades e Institutos de Enseñanza Superior, México, 1966, p. 4. Datos para 1966, cortesía del Instituto Jaliciense de Promoción y Estudios Económicos. La información sobre la Universidad Autónoma de Guadalajara para 1961-1963, del Anuario Estadístico de la UNAM de 1962, 1963 y 1964, pp. 86-87, 125-126 y 129, respectivamente. Datos para 1958-1960 y 1965-1966 son cortesía del Dr. Morales Castro, Universidad Autónoma de Guadalajara. Las cifras sobre el ITESO son cortesía de la Oficina de registro de esta institución. Y los datos sobre la Normal Superior "Nueva Galicia", cortesía de su oficina de registro.

Respecto a la composición de las inscripciones en la educación superior en Jalisco, las ramas científicas y técnicas han incrementado lentamente su participación en el total de inscripciones, de $24.5 \%$ en 1958 a $28.4 \%$ en 1966 . Esta concentración fue todavía menor que la de la República, la cual contaba con $36.3 \%$ del total de inscripciones en estos campos en 1964.15 Dentro de las carreras técnicas y científicas en Jalisco, al igual que la República, la ingeniería mantuvo una posición predominante, aunque cedió un poco de terreno a las ciencias químicas y biológicas y también a la nueva carrera de agronomía. Los dos últimos campos comprenden el $6.9 \%$ y el $2.6 \%$, respectivamente, del total de inscripciones en 1966.

Recíprocamente, las carreras no técnicas ni científicas (definidas zara incluir las ciencias médicas) redujeron su participación relativa en el total de inscripciones de $75.5 \%$ a $71.6 \%$ entre 1958 y 1966, Io que se compara con la cifra correspondiente de $63.7 \%$ para el paîs en

15 Víctor L. Urquidi y Adrián Lajous Vargas, Educación superior, ciencia y tecnología en el desarrollo económico de México: estudio preliminar. México, E1 Colegio de México, 1967, p. 56. 
1964.16 La carrera de medicina acusó el descenso más fuerte, de $23.8 \%$ a $20.5 \%$, pero retuvo su posición como líder en inscripciones. Arquitectura, otro campo tradicionalmente popular en Latinoamérica, ha reducido su participación de $6.1 \%$ a $3.1 \%$. Filosofía y letras también ha sufrido una reducción desde $3.0 \%$ a $1.5 \%$. Administración de negocios y contabilidad han sufrido una ligera reducción, de $19.5 \%$ a $18.2 \%$, mientras que odontología permanece estática en $3.6 \%$. Por otro lado, las inscripciones en la escuela de Derecho se han incrementado de $12.8 \%$ a $16.4 \%$. El hecho de que tal aumento pueda ocurrir ante la presencia de una superabundancia de abogados en Jalisco, al mismo tiempo que está ocurriendo un movimiento gradual hacia las carreras técnico-científicas, tal vez constituye un hecho notable. Una explicación de esta tendencia ciertamente descansa en el bajo costo de la expansión de la inscripción en Derecho, donde no se exigen gastos en laboratorio y equipo. Además de esta ventaja de costos respecto a otros campos, la preferencia tradicional por este campo de estudio no ha sido desintegrada por los cambios dinámicos que ahora están ocurriendo en Jalisco. Economía también ha incrementado su participación de $4.0 \%$ a $4.2 \%$, pero este pequeño cambio refleja principalmente la apertura de esta carrera en la Universidad Autónoma de Guadalajara en 1964. En resumen, hubo cierta indicación alentadora de un incremento en las carreras técnicas entre 1958 y 1966 en el estado de Jalisco, pero el progreso en esta dirección aun deja mucho que desear en comparación con el de la República.

Por desgracia, no fue posible encontrar información satisfactoria sobre las inscripciones anuales para los años subsecuentes en las diversas escuelas y por este motivo no fue posible calcular las tasas de deserción escolar en la educación superior de Jalisco. Sin embargo, se cree que estas tasas fueron muy elevadas. Un indicador de este problema es la muy alta proporción de estudiantes inscritos en el primer año, en oposición a las de los últimos años, para cualquier año académico dado. Las cifras disponibles para 1964 muestran que en la Universidad de Guadalajara, el $32 \%$ del total de las inscripciones estaba concentrado en el primer año y únicamente el $9 \%$ en el último año. En la Universidad Autónoma de Guadalajara las cifras correspondientes fueron $36 \%$ y $12 \%$; en el ITESo 46 y $11 \%$ y en la Escuela Normal Superior "Nueva Galicia", $34 \%$ y $17 \%$.

A continuación se dan unas cuantas observaciones subjetivas generales (desde el punto de vista de una persona educada en los Estados Unidos, pero con alguna experiencia como maestro en Jalisco) concernientes a la calidad de la educación superior en Jalisco:

1) La elevada proporción de estudiantes que trabajan a tiempo completo o que tienen ocupaciones de cerca del tiempo completo obstaculiza la realización del trabajo académico.

2) El alto grado de actividad política en la Universidad de Guadalajara disminuye similarmente el tiempo dedicado al esfuerzo académico (como ejemplo de ta! actividad, el director de la escuela de Economía fue destituido por la presión de los estudiantes en el otoño de 1966). 
3) Falta enfatizar la preparación de trabajos escritos y otras formas de autoexpresión, lo cual proviene de la pobreza de la educación en el nivel de preparatoria.

4) Tanto los estudiantes como los profesores (especialmente los de tiempo completo) permanecen demasiadas horas en el salón de clases y no tienen suficientes incentivos (ni siquiera se espera que los tengan) para realizar una mayor cantidad de trabajo académico fuera de clase.

5) La preponderancia de profesores pobremente pagados de medio tiempo que con frecuencia se encuentran desconectados de los progresos académicos contemporáneos, resulta generalmente en una mala dirección de sus estudiantes y en clases poco atendidas, y también en una falta de investigación académica.

6) Las facilidades de biblioteca son invariablemente inadecuadas y las facilidades en laboratorios y salones de clase lo son algunas veces.

7) El resultado de las deficiencias en la educación superior en Jalisco (que son comunes a muchos países en desarrollo) es frecuentemente un bajo nivel en la calidad de los graduados profesionales en varios campos. Este punto de vista fue confirmado en las entrevistas que se hicieron en varias empresas mediante una muestra llevada a cabo por el autor y también en un estudio reciente de dos expertos alemanes en educación, los que encontraron que únicamente un cuarto o un quinto de los graduados de las escuelas profesionales en México están preparados adecuadamente para el ejercicio de su profesión. ${ }^{17}$

Aunque todos estos comentarios podrían ser más elaborados, son suficientemente familiares y descritos adecuadamente en otra parte como para no prolongar más esta exposición. La Universidad Autónoma de Guadalajara reconoce algunos de estos problemas y está trabajando, bajo su nueva reorganización, para eliminarlos o reducirlos tan rápidamente como sea posible. El ITEso también ha reportado que está manteniendo un programa de estudios que se encuentra sobre el promedio de calidad.

Resumen sobre educación. Harbison y Myers han desarrollado un índice compuesto que señala el nivel general de desarrollo de los recursos humanos en una economía. Este índice se calcula multiplicando el porciento del grupo de edad respectivo inscrito en educación superior por un peso de 5 y agregando el porciento del grupo de edad pertinente inscrito en el nivel medio de educación. El cuadro $10 \mathrm{com}$ para los índices derivados de este modo para varias entidades de México en 1960. Jalisco, en ese año, con un índice de 21.2, mantuvo una posición un poco adelante de los estados relativamente atrasados como Oaxaca (4.2), pero muy detrás de áreas avanzadas como Nuevo León (61.9) y por supuesto el Distrito Federal (101.1). Jalisco se mantuvo también un poco abajo del índice nacional (26.2) aunque excedió el índice nacional eliminado el Distrito Federal (13.0). De acuerdo

17 Véase "Reiteran que es sumamente bajo el rendimiento del estudiante", periódico El Occidental, Guadalajara, junio 20, 1967, p. B-1. 
con la catalogación de los índices de Harbison y Myers para 75 países en 4 categorías, Jalisco caería en la mitad de la tercera clasificación más avanzada, en el área de nivel II, mientras que Nuevo León caería dentro del segundo grupo más avanzado y el Distrito Federal en la categoría más avanzada. Este índice sumario confirma el análisis anterior de los diferentes niveles de educación, el cual muestra que Jalisco aunque progresa más o menos rápidamente en el desarrollo educativo, permanece aún considerablemente a la zaga de otras regiones del país a las que aspira igualar o sobrepasar.

\section{Cuadro 10}

ÍNDICE COMPUESTO HARBISON-MYERS DE DESARROLLO EDUCACIONAL PaRa JALISCO Y otras Áreas DE MÉxico, 1960 *

\begin{tabular}{lrr}
\hline Area & $\begin{array}{c}\text { Valor del } \\
\text { Indice en 1960 }\end{array}$ & NiveI \\
\hline Jalisco & 21.2 & II \\
Nuevo Ie on & 61.9 & III \\
Distrito Federal & 101.1 & IV \\
Oaraca & 4.2 & I \\
RepabIica & 26.2 & II \\
Reprbjica menos & 13.0 & II \\
Distrito Federal & 1.0 & \\
\hline
\end{tabular}

Fuentes: El índice para Jalisco se calculó con datos de los cuadros 6.10 y 6.20 del Anuario Estadístico. Los índices para otras áreas de C. N. Myers, Education and National Development in Mexico, cuadro 15, p. 109.

* El índice compuesto Harbison-Myers es calculado multiplicando el porciento del grupo de edad apropiado inscrito en el nivel de educación superior por un peso de 5, y agregando el porciento del grupo de edad apropiado registrado en educación media.

El cuadro 11 muestra la proporción del presupuesto total del estado dedicado a educación en Jalisco y Nuevo León de 1952 a 1962. Esta información explica parte de la discrepancia en el progreso educativo durante los últimos dos años en estos dos estados. A partir de 1955, Nuevo León empezó a ejercer un intenso programa de desarrollo educativo y consecuentemente empleó una proporción muy alta (casi el $50 \%$ ) de sus recursos presupuestales en este campo. En comparación, Jalisco se encuentra atrasado aunque también se está esforzando por incrementar la participación de sus recursos en la educación (36.4\% en 1962). Por supuesto, los gastos federales son extremadamente importantes en el desarrollo de la educación a nivel estatal, pero suponiendo que una cantidad más o menos proporcional fue gastada por el gobierno federal en Jalisco y Nuevo León durante este período, es claro que el esfuerzo extra a nivel estatal resultó un factor clave en el desarrollo educativo más rápido de Nuevo León. Desafortunadamente no se dispuso de una división detallada de la asignación de esos gastos en educación.

Adiestramiento en el trabajo. Dado que los programas de educación vocacional en Jalisco no están altamente desarrollados, uno podría esperar encontrar un gran uso por el industrial de programas de entrenamiento en las propias empresas para ocupaciones de niveles intermedios y bajos. Éste parece ser el caso de acuerdo con los re- 
Cuadro 11

COMPARACIÓN DE LA PROPORCIÓN DEL PRESUPUESTO DEDICADO A EDUCACIÓN EN JALISCO Y NuEVo LEÓN, 1952-1962

(En porcientos)

\begin{tabular}{llllllllllll}
\hline & 1952 & 1953 & 1954 & 1955 & 1956 & 1957 & 1958 & 1959 & 1960 & 1961 & 1962 \\
\hline Jalisco & 23.3 & 20.1 & 28.6 & 26.7 & 34.2 & 27.3 & 24.3 & 30.0 & 32.7 & 31.2 & 36.4 \\
Nuevo León & 28.1 & 10.9 & 10.4 & 42.4 & 44.3 & 44.2 & 47.1 & 46.3 & 48.0 & 49.3 & 49.4 \\
\hline
\end{tabular}

Fuentes: Anuario Estadístico, edición y referencia de páginas como sigue: 19521954, Anuario Estadístico, 1954, cuadro 63, pp. 152-154; 1955-1957, Anuario Estadistico, 1957, cuadro 67, pp. 184-186; 1958-1959, Anuario Estadístico, 1958-1959, cuadro 82 pp. 148-150; 1960-1961, Anuario Estadístico, 1960-1961, cuadro 6.2, p. 179; 1962, Anuario Estadístico, 1962-1963, cuadro 6.10, p. 182.

sultados obtenidos en una encuesta (hecha por el autor). De las 37 firmas entrevistadas en la industria manufacturera, $33(90 \%)$ tienen una especie de programa de adiestramiento en el trabajo en una categoría de ocupaciones más o menos amplia, y cerca del $80 \%$ han tenido programas de adiestramiento más o menos eficientes para desarrollar obreros calificados. Estos últimos programas consisten casi exclusivamente en varias formas de aprendizaje o de adiestramiento mientras se presta servicio. La mayor excepción a esta generalización es la práctica que siguen algunas empresas de enviar sus trabajadores al Centro Jalisciense de Productividad para pequeños cursos sobre diferentes materias con algunas semanas de duración. Estos cursos están frecuentemente orientados hacia las relaciones humanas, más que a las habilidades vocacionales. El entrenamiento sobre una base más formal, como lo es muy común en los Estados Unidos todavía no se practica ampliamente en el estado de Jalisco. Aunque los programas de adiestramiento de diversas clases fueron instituidos para otras categorías ocupacionales, el número total de tales programas apenas alcanza a igualar el número en la categoría de trabajadores especializados. El siguiente más amplio tipo de programas fue para supervisores y capataces - cerca del $25 \%$ de las empresas entrevistadas proporcionaban adiestramiento para este tipo de empleados. Es muy interesante notar que muchos de los entrevistados expresan cierta queja en el sentido de que ellos se han visto precisados a adiestrar a sus propios trabajadores (trabajadores calificados especialmente). A menudo manifestaron que consideraban que esto era obligación del gobierno aun en casos donde las habilidades de cierto grado de especialización eran particulares a la industria en que ellos se encontraban participando. La impresión fue que la mayoría de los dirigentes de esas empresas no tienen un concepto claro de la relación beneficiocosto de adiestrar a sus trabajadores. ${ }^{18}$

18 Dadas las limitaciones de espacio, no es posible discutir aquí otros aspectos del desarrollo de los recursos humanos que fueron considerados en el estudio completo. Estos otros aspectos incluyen los programas de alfabetización, los efectos de la migración sobre los recursos humanos, la estructura de incentivos, los centros especiales de adiestramiento y las prácticas de contratación de los empleadores. La exclusión aquí no significa que estos elementos deberían o podrían ser legítinamente ignorados por cualquiera interesado en formular un programa 


\section{NECESIDADES DE MANO DE OBRA E IMPLICACIONES EDUCATIVAS}

Pnoyecciones y tendencias recientes. Para determinar los requerimientos de màno de obra en Jalisco implicados en las proyecciones de empleo examinadas anteriormente, es necesario determinar qué estructura ocupacional es adecuada para corresponder al empleo total en cada sector económico. Esto lo hice principalmente a través de un examen de las tendencias pasadas en el avance ocupacional de cada sector y en una serie de entrevistas con empresas del área de Guadalajara, para tener una idea del modo en que las tendencias futuras pudieran derivarse del pasado.

Tal vez el aspecto más interesante de esta fase del estudio se relaciona con las tendencias proyectadas de la mano de obra de alto nivel: trabajadores profesionales, técnicos y administradores. En Jalisco, esta categoría de fuerza de trabajo, que es la categoría clave en el desarrollo de los recursos humanos de cualquier área, quedó muy por detrás de las áreas más desarrolladas de la República en 1960. En ese año, el censo registraba únicamente el $4.6 \%$ de la fuerza de trabajo de Jalisco en la categoría de alto nivel contra un $6.3 \%$ en Nuevo León, $6.2 \%$ en Baja California y un $10.5 \%$ en el Distrito Federal. Sin embargo, Jalisco se encuentra a la cabeza de los estados atrasados como Zacatecas $(2.1 \%)$ y Chiapas $(1.9 \%)$. Se proyecta que esta tasa llegará al $7.2 \%$ para 1980 en el estado de Jalisco, con la mayor parte del incremento concentrado en los grupos "profesionales" y "técnicos" en lugar de la más lenta expansión de la categoría de los administradores.

Al relacionar el patrón de empleo proyectado por estructura ocupacional con los insumos de educación necesitados para producir trabajadores que llenen aquellas ocupaciones, se puede programar la expansión del sistema educativo necesaria para llevar a cabo la tasa de crecimiento esperada de la economía. La relación entre ocupación y calificación educativa no es rígida, y esto se tomó en consideración en los cálculos. Las bases para determinar la calificación educativa de una categoría ocupacional fueron, principalmente, una muestra del censo de problación del año de 1960 que fue proporcionada por El Colegio de México, así como las mencionadas entrevistas con algunas empresas del área de Guadalajara. En general, la proyección supone que las nuevas personas que entran en una categoría ocupacional durante el período previsto necesitan más educación que aquellas que se encuentran ocupando actualmente esos puestos. Sin embargo, sería poco realista suponer que todos estos nuevos miembros de la fuerza de trabajo serán capaces de obtener una preparación satisfactoria, lo cual se toma en cuenta en las proyecciones.

La producción requerida del sistema educativo se muestra en el cuadro 12.

Esta producción requerida permite un cálculo aproximado de las inscripciones necesarias en varios niveles educativos durante los períodos previstos. Empezando con el primer nivel, el cuadro 12 nos indica que se requiere un promedio de producción anual de cerca de 52623

racional de desarrollo de recursos humanos. En la práctica un programa de este tipo debe considerar muchos de estos aspectos si desea evitar redundancias e inconsistencias. 
Cuadro 12

Producción DEL SISTEMA EDUCACIONAL NECESARIO PARA SATISFACER LOS REQUiSTTOS DE FUERZA DE TRABAJO EN JALISCO, 1960-1980

\begin{tabular}{cccc}
\hline $\begin{array}{c}\text { Años de } \\
\text { educación }\end{array}$ & $\begin{array}{c}\text { Producción requerida } \\
\text { del sistema } \\
\text { educacional* }\end{array}$ & $\begin{array}{c}\text { Producción } \\
\text { requerida } \\
\text { acumulada*a }\end{array}$ & $\begin{array}{c}\text { Promedio anual de } \\
\text { producción requerida }\end{array}$ \\
\hline $16+$ & 25201 & 25201 & 1260 \\
$12-15$ & 27332 & 52533 & 2627 \\
$10-11$ & 68161 & 120694 & 635 \\
$7-9$ & 165531 & 286225 & 14311 \\
$4-6$ & 766225 & 1052450 & 52623 \\
$0-3$ & 766 225 & 1052450 & 52623 \\
\hline
\end{tabular}

* Se refiere al número de estudiantes que terminan sus estudios en ese nivel educativo.

$* *$ Incluye el número de estudiantes requerido que terminan y los que continúan en los niveles educativos superiores.

estudiantes (incluyendo los que ya terminaron y aquellos que aún continúan en la escuela secundaria) del cuarto, quinto y sexto grado. Para obtener este producto, se requiere un promedio anual de matrícula en el primer año de cerca de 250000 estudiantes. Esto está basado en un examen de las relaciones pasadas entre el número de estudiantes matriculados en el primer año y el número subsecuente de los que completan satisfactoriamente cuatro, cinco o seis años de educación, tomándose en cuenta la continuación de una tendencia favorable en la deserción. Naturalmente, un juicio o una meta más optimista en relación con la deserción futura reduciría las inscripciones iniciales requeridas. En 1963 la matrícula en el primer año alcanzó únicamente un nivel de 167231 estudiantes; se ve claramente que es necesario hacer un gran esfuerzo en el nivel primario, haciendo más accesible la inscripción en las escuelas a un mayor número de alumnos y reduciendo la proporción de aquellos que abandonan la escuela durante los primeros cuatro años.

Trasladándonos hacia el nivel secundario básico puede verse en el cuadro 12 que se necesita una producción media anual de 14311 estudiantes (de nuevo tomando en cuenta los que terminan y los que aún continúan como estudiantes) del 7\% al 9\% grado. Aunque aquí la información es poco confiable, se estima que para tal producción se necesitará un promedio anual de inscripciones en el primer año de cerca de 20000 estudiantes. La falta de datos de inscripciones por año hace difícil determinar con exactitud las cifras en las inscripciones en el primer año, pero parece que actualmente este nivel educativo no representa un nudo de obstrucción. Esta aseveración está basada primordialmente en la tendencia de las inscripciones totales a este nivel. Por otro lado, la expansión rápida de las inscripciones en este sector refleja una falta relativa de selección a ese nivel, esto es, aquí no existe un proceso formal de segregación como es característico de la mayoría de los sistemas educativos europeos.

Respecto al nivel secundario avanzado (preparatoria y normal), el cuadro 12 señala una media anual de producción (de los que terminan y los que aún continúan) de 6035 estudiantes. Como la deserción a este nivel es menor que en los otros niveles considerados, una inscrip- 
ción anual en el primer año de cerca de 9000 estudiantes es suficiente para obtener la producción requerida. Sin embargo, como actualmente la inscripción en el primer año se aproxima más o menos a la mitad de esta cifra (los datos impiden hacer una determinación exacta), este nivel de educación puede significar un obstáculo durante el periodo previsto.

Finalmente, la producción anual requerida al nivel de educación superior es de cerca de 2627 personas por año en los primeros cuatro años (esto proporcionaría el volumen de subprofesionales requeridos) y 1260 personas de los últimos años (egresados). Se estima que la realización de estas metas, suponiendo únicamente un ligero descenso de la deserción, requiere una inscripción en el primer año de cerca de 5000 alumnos. Esta cifra toma en cuenta el considerable número de estudiantes de otros estados de la República que asisten a las universidades de Jalisco pero que regresarán a sus entidades de origen después de haber terminado sus estudios. De nuevo la información es inadecuada en relación con las cifras actuales, pero aparentemente las cifras de inscripción en el primer año en 1964 fueron ya cercanas a 5000 alumnos y la inscripción en el último año fue casi de 1000 estudiantes. De aquí que este nivel educacional probablemente no constituya un serio obstáculo - al menos desde el punto de vista cuantitativo.

El resumen anterior de los requisitos de inscripción proyectados en ningún caso debe ser considerado como preciso. Más bien, la intención es dar una idea general del orden de magnitud involucrado y las causas más probables que originan los problemas. Un conjunto más exacto de estimaciones requeriría mejor información sobre inscripciones por grados (sobre el nivel primario) y mejores estimaciones de las tasas futuras de deserción escolar. La magnitud de las tasas de deserción estimadas dependerá de que se incremente el número de egresados en los diversos niveles educativos fundamentalmente por medio de inscripciones o bien por menor abandono.

En conclusión, no obstante que el sistema educativo de Jalisco se encuentra a la zaga de otras áreas relativamente avanzadas de la $\mathrm{Re}-$ pública, el mantenimiento de rápidas tasas de expansión en años recientes puede ser suficiente para cubrir los requisitos de mano de obra de la economía. La principal excepción a esta generalización está en las escuelas primarias, que deberán crecer a una tasa mucho más rápida a fin de evitar una deficiencia grave. Por supuesto, se debe tener en cuenta que dicha expansión acelerada aumentará eventualmente la presión por más escuelas en el nivel secundario, independientemente de los requerimientos de mano de obra de la economía.

\section{Problemas específicos de mano de obra en Jalisco}

Esta sección está destinada principalmente a señalar algunas ca racterísticas de la situación de la mano de obra en las industrias de manufacturas y de construcción en Jalisco. La investigación se concentró en estas industrias porque son esenciales al crecimiento industrial de Jalisco, y sus problemas son por lo tanto de gran importancia. También fueron investigados tres hoteles para tener una idea super- 
ficial de los problemas de mano de obra en la dinámica industria turística de Jalisco. Los comentarios son obviamente para dar una idea impresionista más que una imagen precisa de la situación. Los entrevistados fueron por lo general administradores de alto nivel dentro de sus empresas, $y$, en vista de la naturaleza no pecuniaria de las preguntas, generalmente pareció que dieron respuestas directas y sinceras.

En la categoría de personal profesional, el $45 \%$ de las empresas manufactureras entrevistadas manifestó que o ya tienen serios problemas -cualitativos, cuantitativos o ambos-o esperan tener tales problemas dentro de pocos años. La mayoría ( $58 \%$ ) de los problemas indicados fueron de naturaleza cualitativa, por ejemplo, insatisfacción con el trabajo hecho por las personas que ahora trabajan a nivel profesional dentro de la empresa. Casi invariablemente, esto fue atribuido a la preparación imperfecta de estas personas en las universidades. Por el lado cuantitativo, aproximadamente el mismo número de firmas manifestó una escasez o que esperaban tener una escasez de químicos y de ingenieros en los próximos años. Los entrevistados no manifestaron problemas graves respecto a intelectuales sin empleo, e indicaron que, al menos en el presente, no existen graves desequilibrios cuantitativos en la educación superior de Jalisco.

Una proporción más baja de empresas manufactureras (32 \%) manifestó problemas con el personal administrativo de alto nivel. De nuevo, una mayoría ( $55 \%$ ) de los problemas mencionados fueron de naturaleza cualitativa. En el lado cuantitativo, muchos de los entrevistados indicaron una escasez de graduados en administración de negocios. Aunque no lo expresaron muy a menudo, algunos de los entrevistados opinaron que se tiene demasiada confianza en utilizar a miembros de su familia para que ocupen los puestos claves, en detrimento de la eficiencia administrativa.

A nivel subprofesional y técnico, el $32 \%$ de las empresas manufactureras acusó problemas, de los cuales el $53 \%$ fue de naturaleza cualitativa. En muchos casos los profesionales están al parecer llevando a cabo un trabajo que podría ser hecho más apropiadamente por una persona con menos adiestramiento formal. Sin embargo, probablemente esto refleja la mala calidad de la educación formal así como la escasez de técnicos. En este caso, la solución obtenida parecería ser una de tipo pragmático desde el punto de vista de la empresa, aunque no sería óptima desde el punto de vista social, debido al alto costo de proporcionar educación formal avanzada a los llamados profesionales.

En la categoría que incluye los trabajos administrativos de bajo nivel, vendedores y trabajadores calificados de oficina, únicamente el $19 \%$ de las empresas manufactureras manifestó que ha tenido problemas (o los preveía). La mayoría de los problemas mencionados están relacionados con la baja calidad de preparación de las secretarias en las escuelas comerciales.

E1 $49 \%$ de las empresas manufactureras informó que la categoría ocupacional de supervisión era problemática. Una vez más, los problemas cualitativos predominaron (59\%). La queja más frecuentemente citada, por cierto, fue la incapacidad para afrontar problemas humanos en supervisión más que deficiencias del conocimiento técni- 
co requerido. Esto parecería conducir a poner mayor énfasis en la preparación general en las escuelas dejando el conocimiento vocacional específico para que sea obtenido en las empresas a través del adiestramiento en el trabajo.

El mayor problema en esta área fue encontrado en la categoría de trabajadores calificados, donde el $81 \%$ de las empresas manufactureras manifestó tener problemas específicos. Aquí la división entre problemas cuantitativos y cualitativos citados fue más o menos igual. Las quejas cualitativas generalmente se concentraron en la falta de responsabilidad de los trabajadores (embriaguez, etc.) más que en sus capacidades técnicas. Por otra parte, los problemas cuantitativos reflejaron frecuentemente la convicción de que el gobierno debe proveer trabajadores mejor entrenados (a través de escuelas vocacionales) en lugar de esperar que las empresas particulares proporcionen el adiestramiento en el trabajo. No obstante, muchas de estas firmas, por necesidad, entrenan a sus trabajadores. La conclusión alcanzada fue que esta solución es generalmente satisfactoria desde un punto de vista pragmático. Sin embargo, una solución aún más satisfactoria sería indudablemente factible si las empresas privadas aceptaran esta función de entrenamiento como una de sus funciones normales, facilitando una mejor organización a tales programas. Respecto del alegado nivel alto de irresponsabilidad de los trabajadores, parece claro que la relativamente baja escala de salarios para los trabajadores especializados contribuye fuertemente a dicho comportamiento.

Al nivel no calificado, como debería esperarse, los problemas cuantitativos no fueron de importancia. El mayor problema cualitativo a este nivel parece ser una vez más la "irresponsabilidad", que se debe más bien a la falta o ausencia de educación.

Con respecto a la relación entre los programas de investigación y las necesidades de mano de obra, únicamente cerca de una tercera parte de los programas manufactureros se ocupaban en alguna actividad de esta naturaleza, y entre los que lo hacían el número promedio de personas ocupadas fue de únicamente 2.5. Por esto parece improbable, a pesar de los programas, generar una fuente importante de demanda de mano de obra calificada en el período previsto.

La situación en la industria de la construcción, en la cual sólo fueron entrevistadas tres firmas, no parece diferir de manera importante de la industria manufacturera. La mayoría de los problemas citados se encontró indistintamente en las categorías de profesionales y trabajadores especializados, aunque en esta última fue demostrada una mayor satisfacción en cuanto a la calidad de los trabajadores. En los tres hoteles entrevistados predomina el problema de los trabajadores especializados. La escuela vocacional propuesta en Guadalajara para trabajadores de la industria turística deberá aliviar esta situación.

Al final de las entrevistas se hicieron algunas preguntas generales. En respuesta a la pregunta: "¿cuál es su problema más grave por lo que respecta al trabajo o a la fuerza de trabajo?", el $48 \%$ de las empresas manufactureras indicó la baja calidad o la falta de responsabilidad de los trabajadores (incluyendo profesionales). Otro $25 \%$ mencionó la escasez de trabajadores especializados. Cerca del $59 \%$ de las empresas manufactureras indicó que emplean trabajadores no es- 
pecializados provenientes de las áreas rurales, lo que indica la importancia de dotar a estas personas de escuelas para que los suburbios de las ciudades no estén sobrepoblándose con trabajadores desocupados y no educados. Únicamente el $30 \%$ de las empresas manufactureras indicó que ellas proporcionan iguales oportunidades (incluyendo la contratación y la promoción) de empleo sin tomar en cuenta el sexo. Sin embargo, las mujeres fueron empleadas en el $81 \%$ de las firmas entrevistadas. Por último, poco más o menos la mitad de las industrias manufactureras entrevistadas respondieron que no contratan trabajadores con más de 35 años de edad.

\section{CONCLUSTONES}

A pesar de las limitaciones inherentes a un estudio de esta naturaleza y aunando a estas limitaciones las dificultades de trabajar con información a nivel estatal, este estudio nos indica que es posible analizar el desarrollo de los recursos humanos con alguna significación, a este nivel, en un país en desarrollo. Se reconoce generalmente que las posibilidades para habilitar un programa de desarrollo de recursos humanos, basado en un estudio como éste, están restringidas por la excesiva confianza que debe ponerse en los gastos del gobierno federal. No obstante, los estudios a nivel estatal pueden ser útiles para guiar los gastos federales hacia los canales educativos apropiados así como para obtener una mejor y más eficiente utilización de los recursos presupuestarios estatales disponibles. Algunos de los resultados específicos más importantes obtenidos en este estudio son los siguientes:

1. Jalisco se ha caracterizado y continuará caracterizándose por una rápida tasa de crecimiento natural de la población (más de $3 \%$ anual), la cual es probable se incrementará por la inmigración de las entidades vecinas relativamente pobres. Este crecimiento rápido de la población tiene el efecto de crear una elevada proporción de población joven. Esta característica demográfica, típica de los países en desarrollo, es una fuente de tremenda presión, en primera instancia en el sistema educativo que debe proporcionar entrenamiento para estos jóvenes, y en segundo lugar, en la economía, la cual debe absorberlos en una forma productiva.

2. Gran parte de este incremento de la población se concentrará en las áreas urbanas -particularmente el área metropolitana de Guadalajara-cualquiera que sea la tasa de crecimiento económico en estas áreas. Esto implica que deberá hacerse un gran esfuerzo fuera del sector agrícola con objeto de poder absorber estos incrementos en forma efectiva. Mucha de esta capacidad de absorción tendrá, indiscutiblemente, que ser generada (ya sea directa o indirectamente) por la expansión del sector manufacturero. Las técnicas relativamente intensivas de capital que serán introducidas en este sector implicarán que los otros sectores no agrícolas tendrán que proveer una alta proporción de nuevos empleos. Por otro lado, el desarrollo continuado del sector agrícola será esencial para el crecimiento de la industria manufacturera orientada hacia la agricultura (una orientación que no parece cambie en forma importante antes de 1980). 
3. El número total de nuevos empleos que será necesario crear para evitar que se eleven aún más los coeficientes de desempleo y subempleo es del orden de una media anual de 38600 ntre los años 1970 y 1980.

4. El desarrollo del sistema de educación en Jalisco está rezagado por un amplio margen respecto al de las áreas más avanzadas de la República, tales como Nuevo León y el Distrito Federal. Esto fue cierto en todos los niveles de educación, pero el desarrollo de la educación primaria parece haber tenido un atraso muy particular. En contraste, las inscripciones en el nivel secundario y de educación superior se han incrementado rápidamente en los años recientes no obstante que este crecimiento está limitado por la producción de egresados del nivel primario.

5. El limitado crecimiento del sistema de educación es notorio tanto en los porcientos de los grupos de edad respectivos inscritos como en las altas tasas de deserción en todos los niveles. Cualquier programa de desarrollo de los recursos humanos que sea formulado deberá enfrentarse a estos problemas fundamentales.

6. El análisis de los requerimientos de mano de obra indica que el sistema educativo, dado su impulso actual, debe estar capacitado para afrontar las necesidades de la economía en los niveles altos de ocupación desde el punto de vista de la cantidad necesaria de graduados. Por otro lado, tal parece que continuarán apreciándose obstáculos sustanciales en algunas categorías de los niveles más bajos de ocupación, particularmente en la de trabajadores especializados y supervisores, a menos que se asigne al desarrollo de la educación primaria mayor prioridad dentro del presupuesto. El crecimiento de la educación primaria debe ser en alguna forma orientado hacia las áreas rurales no obstante la disminución relativa de este sector. Dicha reorientación facilitará el trabajo de absorber a los inmigrantes rurales a la ciudad así como incrementar la productividad agrícola. La acelerada expansión primaria deberá ejercer presión a su-vez en el crecimiento de las escuelas secundarias (muchas de las cuales son operadas por particulares); pero por el momento el nivel primario deberá recibir la mayor prioridad. En el presente, la expansión de la educación secundaria también puede ser complementada en forma muy significativa mediante la expansión de los programas de adiestramiento en el trabajo en la industria privada. Una mayor persuación moral, así como otros métodos, pueden indiscutiblemente ser usados con ventaja por el gobierno para estimular la creación, expansión y mejoramiento de dichos programas en la industria privada. Tal parece que no hay problemas cuantitativos en la educación superior, excepto por la necesidad de continuar y quizás de acelerar la lenta tendencia en dirección de los campos científico y técnicos.

7. Las estimaciones burdas de un promedio anual requerido de inscripciones en Jalisco fueron del siguiente orden de magnitud (todas las cifras se refieren al primer año de inscripción, en ese nivel de educación, el cual es requerido para obtener la producción necesitada de ese nivel en los años subsiguientes): 
Primaria

Secundaria básica

Secundaria avanzada

Universidad
250000

20000

9000

5000

8. No obstante el cuadro cuantitativo moderadamente alentador, salvo en la educación primaria, que ha surgido en este estudio, existen problemas muy graves por el lado cualitativo. En efecto, y a menos de que se hagan revisiones incisivas en la calidad de la educación, gran parte del incremento en la producción cuantitativa de la educación no tendrá el impacto económico necesario. Esto es especialmente cierto para la educación superior pero también para los otros niveles de educación profesional. En la educación superior, la baja calidad de los profesionales graduados resulta frecuentemente en que estos graduados estén trabajando en puestos subprofesionales. Dicha situación implica un serio desperdicio de recursos educativos y puede llevar a un desasosiego por parte de los individuos comprometidos. Una mejor solución sería buscar la forma de lograr una mayor expansión de las carreras subprofesionales en el sistema de educación, aunque esto requeriría mejorar la imagen de dichas carreras con el objeto de atraer estudiantes.

9. Para idear y habilitar un programa efectivo de desarrollo educativo en el nivel primario ( $\mathrm{y}$ en menor grado en nivel secundario) es necesaria una completa reorganización de los departamentos de educación federal y estatal en Jalisco. Actualmente estas oficinas operan sobre una base estrictamente de "día a día", enviando toda la información obtenida a la ciudad de México y en algunas ocasiones ni siquiera tomándose la molestia de guardar sus propias copias, y en general, tienen una capacidad muy discutible para llevar a cabo un programa racional de desarrollo. Aún más, en la actualidad hay muy poca coordinación entre estos departamentos, situación que debe ser rectificada como prerrequisito para llevar a cabo un programa efectivo de desarrollo.

10. Los resultados de este estudio, en particular en relación con la estructura de la ocupación futura y las necesidades de producción educativa, no deben interpretarse como predicćiones o requisitos precisos. En vez de eso, este estudio constituye un primer paso, aunque imperfecto, para colocar una base hacia la formación de un programa efectivo de desarrollo de los recursos humanos en Jalisco. Tal vez el principal valor del presente estudio sea la demostración de la viabilidad y deseabilidad de estudios adicionales de los recursos humanos, especialmente en Jalisco, y en general a niveles regionales y estatales en los países en vías de desarrollo.

\section{APENDICE I}

\section{TÉCNICAS DE ANÁLISIS DE LOS RECURSOS HUMANOS}

En los años recientes, ha aparecido gran cantidad de literatura que trata sobre la relación entre educación y desarrollo económico. Con frecuencia, el análisis se ha ido extendiendo para tocar otros aspectos distintos al de la educación formal que afectan el desarrollo de los recursos 
humanos. Aunque este interés en el desarrollo de los recursos humanos no carece de antecedentes, únicamente en los últimos años ha recibido la atención debida por parte de los economistas y ha sido enfocado de una manera sistemática hacia la contribución al desarrollo de los recursos humanos en el crecimiento económico de los países menos desarrollados. Este surgimiento tardío del interés en los ingredientes humanos del desarrollo económico puede probablemente ser atribuido en gran parte a la concentración keynesiana sobre la inversión como una variable económica clave y al resultante énfasis sobre la acumulación de unidades de capital físico como la clave del crecimiento económico.

La consecuencia de la apertura de este campo nuevo del análisis económico ha sido un intento de la mayor parte de las naciones en desarrollo por incorporar un programa de fomento de los recursos humanos a sus programas globales de desarrollo económico, o al menos en forma adyacente. Aunque la integración de estos dos tipos de programación ha sido hecha sin congruencia, en la mayoría de los casos se ha avanzado a medida que aumenta el reconocimiento, por parte de los planificadores económicos, de la importancia del desarrollo de los recursos humanos.

Han sido formulados un número considerable de enfoques diferentes sobre el análisis de las relaciones entre los recursos humanos y el desarroIlo económico. Muchos de éstos fueron revisados y criticados en el estudio original sobre el que se basa esta monografía. Dos enfoques, sin embargo, han dominado la atención de los investigadores en relación con programas prácticos. El primero, que se ha popularizado en los Estados Unidos y que puede ser caracterizado como el enfoque de "La tasa de beneficio de las inversiones en recursos humanos", implica esencialmente un tipo de análisis de beneficio-costo. Para un ejemplo excelente de la aplicación de este método, el lector puede consultar un estudio de Martín Carnoy, The Cost and Return to Schooling in Mexico: A Case Study (tesis doctoral inédita, Departamento de Economía, Universidad de Chicago, septiembre de 1964).

El segundo enfoque es aquel que se ha utilizado en este estudio y que ha sido llamado método de "requerimientos de mano de obra". Esta técnica fue desarrollada principalmente por los investigadores de la Organización para la Cooperación Económica y el Desarrollo $(O E C D)$ con motivo del Proyecto Regional del Mediterráneo a principios del decenio del 60 . En este proyecto se aplicó el método a varios países de Europa, para cada uno de los cuales la $O E C D$ publicó un estudio por separado.

Esencialmente este método es un tipo de análisis de oferta y demanda de mano de obra. Implica una proyección de la población, y de la fuerza de trabajo para un número dado de años (preferiblemente entre 15 y 20) en el futuro, constituyendo así la oferta cuantitativa de mano de obra. Esto es seguido por una proyección del crecimiento esperado o deseado de la economía por actividad económica (sector). Estas proyecciones no deberán ser interpretadas como un intento para predecir el futuro, lo cual es obviamente imposible, sino para establecer una meta técnica que permita un desarrollo más racional de los programas de recursos humanos. Cuando estas proyecciones económicas son convertidas a estimaciones de empleo por categoría ocupacional resultan en una aproximación de la demanda de mano de obra en el período previsto. De este modo, estas proyecciones económicas y demográficas pueden ser convertidas en la producción requerida del sistema educativo, las cuales a su vez sirven como guía para la programación de las inscripciones en los diferentes niveles de educación y tipos de escuelas, etc. Una exposición más detallada de todo esto puede ser encontrada en el estudio original o en el libro de Herbert Parnes, Forecasting Educational Needs for Economic and Social Development (París, OECD, 1962). 


\section{APÉNDICE II}

\section{SUGERENCIAS PARA INVESTIGACIONES FUTURAS}

Durante la preparación de esta tesis, han aparecido diversos aspectos, los cuales, desde el punto de vista del autor, ameritan una investigación posterior. Algunos de los más sobresalientes se enumeran a continuación:

Se necesita mayor información sobre los patrones de participación femenina en la fuerza de trabajo por grupos de edad en Jalisco (y en todo México). Específicamente, hace falta aún una explicación adecuada para saber por qué estas tasas se elevan desde los 25 años hasta la ancianidad en lugar de ir declinando gradualmente como en otros países en desarrollo.

Se necesitan estudios sobre los patrones de migración en Jalisco, particularmente en lo que se refiere a la acelerada corriente que ha estado llegando de otros estados de la República en los últimos años. De particular interés será necesario un examen de la efectividad del sector agrícola (especialmente de los ejidos) para absorber el crecimiento de la población y el efecto de tal absorción en la productividad de este sector. También, de posible consecuencia será un análisis de las características económicas y de educación de los migrantes que llegan y salen de Jalisco. Un caso especial que podría ser de gran importancia es el de los braceros, los trabajadores emigrantes del campo que en gran número han salido hacia los Estados Unidos en los últimos años. Jalisco es considerado uno de los principales abastecedores de tales trabajadores. Observaciones superficiales apoyarían la hipótesis de que los braceros que regresan son agentes efectivos de cambio en sus comunidades rurales. Esto se debe a los nuevos fondos de capital adquiridos y a su nuevo punto de vista más dinámico obtenido por el hecho de estar expuestos a una economía más altamente desarrollada.

Respecto a la tendencia del incremento natural de la población, sería interesante saber más acerca del conocimiento, actitudes y prácticas de la población de Jalisco -especialmente la de Guadalajara- con respecto a la planificación familiar. Dicha información puede ser comparada con las cifras de estudios relativos hechos en otras áreas de Latinoamérica (incluyendo la ciudad de México), para evaluar la probabilidad de que tales medidas desempeñen un papel importante en el decrecimiento de la fecundidad en Jalisco dentro de los próximos años. Esto será de especial interés en vista de la reputación de Jalisco como un estado conservador y en el cual la iglesia católica juega aún un papel muy importante en la formación de las actitudes.

En el área del empleo serán muy valiosos los estudios futuros acerca de los efectos de la generación de empleos en la industria de Jalisco. Dichos estudios deberán considerar las nuevas oportunidades de empleo generadas dentro de la industria manufacturera, y las relativas a las oportunidades de empleo que puedan aparecer en el comercio, los servicios, etc. En particular, la naturaleza de las tecnologías (intensivas de capital contra intensivas de trabajo) utilizadas en el sector manufacturero en relación con las oportunidades de empleo, es materia de gran importancia que requiere de futura investigación. Otra área de interés es la tendencia de la productividad dentro de los sectores de rápido crecimiento, como lo son el comercio y los servicios (por ejemplo, qué proporción de los nuevos trabajos en estos sectores refleja meramente una transferencia del subempleo de los alrededores de la ciudad y qué proporción representa un incremento real en el empleo).

Información y análisis futuros son necesarios sobre el sistema de educación de Jalisco. La información sobre el nivel secundario es particular- 
mente de poca confianza. Aquí, será muy útil un cuidadoso examen sobre la composición de las inscripciones entre varias clases de escuelas (incluyendo los cursos vocacionales). Mejor información es también necesitada en este nivel sobre los números de inscripciones en cada año con miras a obtener una idea más acertada de la deserción y del número de graduados. Una mayor investigación sobre la calidad de la instrucción que se proporciona en varios niveles de educación también será reveladora aunque esto se adentra en el campo del educador profesional.

Sobre el aspecto de las necesidades de mano de obra, sería útil subdividir la clasificación ocupacional "trabajadores profesionales y técnicos" en dos clases distintas. Esto facilitará el cálculo de los requerimientos de educación que son generalmente más altos para ocupaciones profesionales que para las técnicas. Además, sería valioso subdividir a los trabajadores profesionales (como un mínimo) de acuerdo a una clasificación en técnico-científicos y científicos no técnicos para poder apreciar la dirección deseable de la expansión de la educación superior. Además, deberán hacerse proyecciones especiales sobre la necesidad de algunas ocupaciones profesionales, como médicos, donde la relación con la expansión de la economía es probablemente tenue.

Finalmente, sería conveniente preparar una proyección cuidadosa de las inscripciones y de la producción futuras a los diversos niveles basadas en tendencias anteriores. Dicha proyección no fue tomada en cuenta en este estudio, excepto en una forma muy gruesa, debido a la falta de una base estadística adecuada. Una proyección de esta naturaleza constituirá, en cierto sentido, la demanda de educación por el público en una forma muy distinta a los requisitos de mano de obra de la economía. Una comparación entre estos dos conjuntos de cifras nos mostraría entonces la brecha por cubrir ( si se mantienen las tendencias presentes en el sistema educativo) entre la mano de obra especializada con que se cuenta y la mano de obra especializada que se necesita. Este es un enfoque más satisfactorio que calcular únicamente los que serían requeridos sin relacionar estos totales con las disponibilidades esperadas. 